\title{
Modified Mackenzie Sound Equation and CVOA Algorithm Based UASN Routing in Dissolved Gases with Divergent Wind Environment to Reduce Propagation Delay
}

Amirthavalli R ( $\square$ amirthavallisenthil@gmail.com )

Velammal Engineering College https://orcid.org/0000-0001-8637-8124

NR Shanker

Aalim Muhammed Salegh College of Engineering

\section{Research Article}

Keywords: Underwater Acoustic Sensor Network , GPR , GROM , Mackenzie Sound equation

Posted Date: June 7th, 2021

DOI: https://doi.org/10.21203/rs.3.rs-526618/v1

License: (c) (1) This work is licensed under a Creative Commons Attribution 4.0 International License.

Read Full License 


\title{
Modified Mackenzie Sound Equation and CVOA Algorithm Based UASN Routing in Dissolved Gases with Divergent Wind Environment to Reduce Propagation Delay
}

\author{
Amirthavalli $\mathbf{R}^{1} \odot$. Thanga Ramya . $\mathbf{S}^{2}$. Shanker $\mathrm{NR}^{3}$
}

\begin{abstract}
In Underwater Acoustic Sensor Network (UASN), routing and propagation delay is affected through various water column environment effects in each node such as temperature, salinity, depth, gases, divergent and rotational wind. High sound velocity increases the transmission rate of packets and high dissolved gases in the water environment increase sound velocity. High dissolved gases and sound velocity environment in water column provide high transmission rates among UASN nodes. In this paper, the Modified Mackenzie Sound equation calculates sound velocity in each node for energy-efficient routing. Golden Ratio Optimization Method (GROM) and Gaussian Process Regression (GPR) predict propagation delay of each node in UASN from dataset which consists of Mackenzie Sound equation calculated sound velocity based on temperature, salinity, depth, dissolved gases. Dissolved gases, rotational and divergent winds, and stress play major problems in UASN, increases propagation delay and energy consumption. Predicted values from GPR and GROM lead to node selection and among selected nodes Corona Virus Optimization Algorithm (CVOA) routing is performed. The proposed GPR-CVOA and GROM-CVOA algorithm solves the problem of propagation delay and consumes less energy in nodes based on appropriate tolerant delays in transmitting packets among nodes during high rotational and divergent winds. From simulation results, CVOA Algorithm performs better than traditional DF and LION algorithms.
\end{abstract}

Keywords Underwater Acoustic Sensor Network · GPR · GROM • Mackenzie Sound equation

Amirthavalli R

amirthavallisenthil@gmail.com

Thanga Ramya S

str.it@rmd.ac.in

Shanker N.R.

drnrshanker@gmail.com

1 Velammal Engineering College, Chennai, TN, India

2 RMD Engineering College, Chennai, TN, India

3 Aalim Muhammed Salegh College of Engineering, Chennai, TN, India 


\section{Introduction}

UASN plays a vital role in monitoring and surveillance of ocean areas in various depths. The monitoring and surveillance applications such as pollution monitoring, underwater exploration, seismic exploration, underwater navigation and tracking, hydrography, oceanography, Unmanned Underwater Vehicle (UUV), anti-submarine warfare needs efficient routing algorithms in the different ocean environment and water column variations. The ocean environments are depth, salinity, temperature, and pressure. The water column variations are geometric and Doppler effects, rotational and divergent wind stress, dissolved gases, sedimentation drift. Data transmission in water column variation is a challenging task in UASN, due to frequent variations in the water column such as dissolved gas and rotational and divergent wind stress. Prediction or measurement of the ocean environment and water column parameters need efficient methods. However, algorithms such as VODA [1], RVPR [2], glider-assist routing [3], DVOR [4], DQELR [5], RCAR [6], DSR-SDN [7], EBOR [8] developed for efficient routing in different ocean environmental conditions, whereas for water column variations such as geometric spread, sedimentation drift, Doppler effect few UASN algorithms proposed such as COCAN, LOCAN [9]. In UASN, acoustic signal performs better than extreme Low frequencies (ELF) due to less attenuation in underwater.

Data transmission between underwater sensor nodes depends on the water column, sound speed profile, and dissolved gases in the sea. Data transmission and routing in UASN is influenced by ocean parameters such as temperature, salinity, pressure, and depth of the sea. The pressure in the ocean increases as depth increases, dissolved gases vary according to the depth of the ocean. The dissolved gases are high for low salinity water, higher the pressure leads to higher dissolved gases in seawater. With the decrease in temperature, dissolved gases are higher. The dissolved $\mathrm{CO} 2$ concentration is proportional to sound speed. Apart from $\mathrm{CO} 2, \mathrm{H} 2 \mathrm{~S}, \mathrm{CH} 4$, and $\mathrm{NH} 3$ in water affect data transmission in UASN. Moreover, data transmission varies for different ocean depths due to different concentrations of gases. The speed of sound is very fast for high temperature and low for thermocline regions where the temperature drops. Similarly, the speed of sound is proportional to pressure. The sound travels at a high speed in distilled water compared to ocean water. The high speed of sound in the ocean leads for high data transmission. The Speed of sound depends on ocean temperature. For example, the speed of sound increases by $4 \mathrm{~m} / \mathrm{s}$ with a 1 -degree increase in temperature, such an increase in temperature improves channel bandwidth during data transmission.

\subsection{Problem Statement}

In UASN, data transmission is affected due to water column variations, pressure, depth, salinity, temperature, sound profile, and dissolved gases. Many researchers developed various routing algorithms for data transmission in UASN. However, existing routing algorithms measure pressure, depth, salinity, and temperature, sound profile using the various sensors such as temperature, pressure, depth, salinity, and for sensing the dissolved gases various sensors such as optode, NDIR [10] is used. However, the energy consumption of sensors in the node is more and need an alternative approach for measurement. For example, the optode sensor consumes $1.8 \mathrm{~W}$ of power, and the response time for providing the result is about 3 min. Similarly, delay in data transmission arises underwater due to divergent and rotational wind environments. Nodes in UASN needs efficient algorithm for prediction of ocean parameters such as divergent, rotational wind environment. Furthermore, the number of sensors in nodes needs to be replaced with an empirical method of calculation of dissolved gases underwater and prevent more power consumption in each node. The drawback of the response time of each sensor leads to more delay in UASN, which need to be addressed along with divergent and rotational wind environment. 


\subsection{Contributions}

Nodes in UASN need a smaller number of sensors to reducing battery consumption. For measuring the depth, temperature, pressure, salinity, wind direction, dissolved gas, wind speed, sedimentation drift, Doppler Effect, and geometric spread, and thermocline there are various sensors. These sensors in a single node will consume more energy, responsivity time and delay in packet delivery. To reduce the number of sensors in node and improve the energy efficiency of the node through an empirical approach, the speed of sound in underwater is estimated through the Mackenzie equation. Mackenzie equation replaces sensors such as wind, wind direction, sedimentation drift, Doppler Effect, and geometric spread through the sound profile calculation obtaining from the Mackenzie equation. In this paper, a Modified Mackenzie equation (MME) is proposed for the estimation of dissolved gases. Modified Mackenzie equation replaces sensors in node and estimates parameters of the ocean environment. In UASN, routing based on water column variations especially for divergent and rotational wind environments are yet to be designed. With this as inspiration, this paper proposes a novel routing protocol that takes into account the ocean's parameters like dissolved gases, depth, salinity, temperature, rotational, and divergent wind stress.

i. MME calculates the sound velocity of the node region, which includes dissolved gases. The node in the region needs an average sound velocity of $1450 \mathrm{~m} / \mathrm{s}$ is selected for routing. MME helps to calculate the divergent and rotational wind through sound profile with Weather Observations through Ambient Noise (WOTAN). So, a sensor such as optode, NDIR is never required in each node.

ii. GROM and GPR predict propagation delay of the nodes during the rotational and divergent wind stress in ocean-based on Dataset.

iii. CVOA algorithm is applied for routing in UASN to avoid propagation delay due to dissolved gases, sedimentation drift, and water column variations.

\section{Related Work}

The Table 1 shows different algorithms in UASN and its advantages and disadvantages

Table 1 Literature Survey on different algorithms on UASN

\begin{tabular}{|c|c|c|c|}
\hline [Ref]/Year & $\begin{array}{l}\text { Problem } \\
\text { (UNDER WATER } \\
\text { SENSOR NETWORK) }\end{array}$ & Methodology & $\begin{array}{l}\text { Advantages } \\
\& \\
\text { Disadvantages }\end{array}$ \\
\hline$[11] / 2020$ & $\begin{array}{l}\text { Routing protocol for } \\
\text { 3D UASNs. }\end{array}$ & $\begin{array}{l}\text { Game-theoretic routing } \\
\text { Protocol for forwarding area, } \\
\text { node degree in forwarding area, } \\
\text { game theoretic based } \\
\text { forwarding }\end{array}$ & $\begin{array}{l}\text { Estimates the node degree in } \\
\text { the forwarding area. }\end{array}$ \\
\hline [12]/2020 & Localization. & $\begin{array}{l}\text { Mobile-beacon Based Iterative } \\
\text { Localization }\end{array}$ & $\begin{array}{l}\text { Balanced energy conservation, } \\
\text { Reduced localization error }\end{array}$ \\
\hline [13]/2020 & $\begin{array}{l}\text { Localization - Direct } \\
\text { Position Determination } \\
\text { method }\end{array}$ & $\begin{array}{l}\text { A weighted MUSIC direct } \\
\text { source localization approach }\end{array}$ & $\begin{array}{l}\text { Direct position determination } \\
\text { of nodes }\end{array}$ \\
\hline [14]/2019 & $\begin{array}{l}\text { Trust, energy balance } \\
\text { in the cluster. }\end{array}$ & $\begin{array}{l}\text { Energy-balanced Trust Cloud } \\
\text { Migration scheme (ETCM) }\end{array}$ & $\begin{array}{l}\text { Evaluates the trust value of } \\
\text { nodes }\end{array}$ \\
\hline$[15] / 2020$ & $\begin{array}{l}\text { Low location accuracy } \\
\text { and mobility, high }\end{array}$ & $\begin{array}{l}\text { MPL (movement prediction } \\
\text { location) }\end{array}$ & $\begin{array}{l}\text { Mobile prediction and node } \\
\text { localization }\end{array}$ \\
\hline
\end{tabular}


consumption of energy

of the nodes and

communication

overhead

[16]/2019 Trust model

$[17] / 2020$

Route interruptions and packet collisions.

Propagation delay and Localization of nodes under different water current parameters.

[19]/2020 Energy efficient and data collection.

[20]/2019 Hybrid attacks of channel jamming and eavesdropping.

[21]/2018 MAC protocol avoid collision

$[22] / 2019$

[23]/2020

[24]/2020

[25]/2021

$[26] / 2020$

[27]/2019

$[28] / 2020$
Scalable networking protocol

Delay in Data collection

MAC design
Maximize lifetime of network

Underwater acoustic channel estimation

Trust model for Underwater node communication Localization accuracy
Synergetic trust model based on SVM (STMS)

Collision and interruption tolerant protocol (CITP): integrated routing-MAC design - adaptively forms the route

\section{Cooperative location-aware network}

Provides cluster-based network and

Optimal Cluster head based on energy efficiency

A bandit-based hybrid attack.

MC-UWMAC protocol: control channel with grid-based slot assignment and 2-hop conflictfree data channel, a quorumbased data channel allocation procedure is used

Most energy-efficient transmission rate (MEETR)

Static-dynamic discriminative compressed sensing (SDD-CS)

iTrust model based on isolation forest algorithm

Localization with a Mobile Beacon via Motion

Compensation in Underwater

Sensor Networks

JOIN protocol to optimize the framework

Prediction-based delay optimization data collection algorithm (PDO-DC) based on Kernel Ridge Regression (KRR)

MAC-based on Probabilistic SDMA - An Angle of Departure (AOD)-based solution for coarse interval estimation and estimation via unscented Kalman filtering

Wireless recharge through ultrasonic waves
Trust evaluation through Machine learning such as SVM and k-means

Routing on the fly bypassing the interrupted links

Eliminates the problems of the asynchronous clock, stratification effect, and mobility of nodes

Data Collection delay is decreased.

Novel feedback observation method, A virtual expert, a self-detection step to keep away from the traps

Collision-free data communications of both control channel and data channels

Performs only for Time Division Multiple Access Models the hybrid channels to static and time-varying components

Detection accuracy in environmental noise such as interference of channels

Improved accuracy and reliability

Network disruption due to collision, when a new node joins the network

Data collection speeded up by visiting fewer clusters

Reduces interference and retransmission probability

Covers longer distances 


\begin{tabular}{|c|c|c|c|c|}
\hline$[30] / 2020$ & $\begin{array}{l}\text { 3D UASN cost } \\
\text { effective network } \\
\text { design }\end{array}$ & 2 und & ted ALOHA MAC & $\begin{array}{l}\text { Computational complexity is } \\
\text { reduced ARQ } \\
\text { transmission probability } \\
\text { and the average } \\
\text { queueing delay of an } \\
\text { underwater sensor is } \\
\text { improved. }\end{array}$ \\
\hline Proposed & $\begin{array}{l}\text { Rotational and } \\
\text { divergence wind, } \\
\text { dissolved gases in } \\
\text { ocean, \& water column } \\
\text { variation }\end{array}$ & $\begin{array}{l}\text { (i) } \\
\text { (ii) } \\
\text { (iii) }\end{array}$ & $\begin{array}{l}\text { Modified Mackenzie } \\
\text { Sound Equation } \\
\text { measures sound } \\
\text { velocity } \\
\text { WOTAN measures } \\
\text { rotation and divergent } \\
\text { wind stress } \\
\text { GROM and GPR } \\
\text { predict propagation } \\
\text { delay }\end{array}$ & $\begin{array}{l}\text { Propagation delay improves } \\
\text { in dissolved gases, } \\
\text { sedimentation drift, and water } \\
\text { column variations } \\
\text { environment. }\end{array}$ \\
\hline
\end{tabular}

\section{Methodology}

In recent years, routing algorithms are proposed for UASN based on location-based and depth-based. These routing algorithms never consider physical properties such as dissolved gases and effects of sound velocity during data transmission underwater. Sound velocity in dissolved gases plays a vital role in underwater communication for efficient data transmission. In this paper, Modified Mackenzie Sound Equation based sound velocity measurement, Gaussian process regression based CVOA (GPR-CVOA), and Golden Ratio Optimization Method Based CVOA (GROM-CVOA) Algorithm Routing in Dissolved Gases with Divergent Wind Environment for Reducing Propagation Delay are proposed. The proposed GPR-CVOA and GROM-CVOA consider underwater parameters such as temperature, depth, salinity, pressure, along with major underwater parameters such as sound velocity, rotational, divergent wind, and dissolved gases for routing and avoids propagation delay. For calculating sound velocity, the modified Mackenzie equation uses the above underwater parameters. The traditional Mackenzie equation considers only depth, salinity, and temperature, whereas the proposed modified Mackenzie equation in this paper, includes parameters such as dissolved $\mathrm{CO} 2$ and computes acoustic velocity. Acoustic velocity is predicted for each node in the network for knowing environment behaviors and estimates propagation delay through GPR or GROM due to dissolved gases and acoustic velocity underwater. The nodes in a high acoustic velocity environment are selected for data transmission. In each node, the modified Mackenzie equation calculates sound velocity for every 30 minutes and updated in each node database in UASN. The node database consists of the following sensor-measured parameters such as temperature, dissolved gases, depth, and salinity and estimated parameters such as sound velocity, rotational wind stress, and divergent wind stress. The Sound velocity measure through a modified Mackenzie equation. Through Sound velocity, rotational, divergent wind stress measure with Weather Observations through Ambient Noise (WOTAN) estimation equation as discussed in the following section [31]. Each node database is collected and applied in GPR or GROM for identification of propagation delay of each node based on the water column environment. The propagation delay is estimated for each node and stored in each node database every 30 minutes. Nodes with less propagation delay are selected for routing by the CVOA algorithm. However, propagation delay changes among the selected nodes, and such changes are handled through delay-tolerant method of transmitting data in UASN. Fig. 1 shows Modified Mackenzie Sound Equation, Gaussian process, and Golden Ratio Optimizations Based CVOA Algorithm Routing implementation in UASN. 


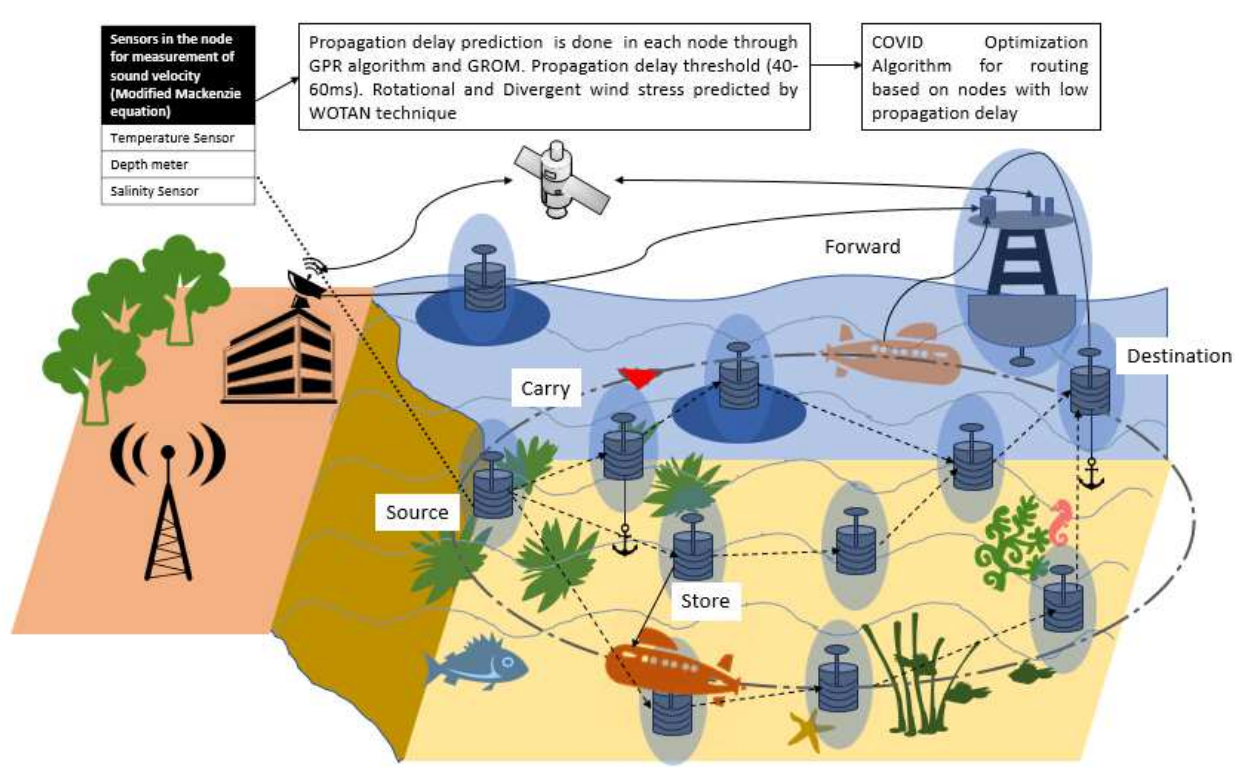

Fig. 1 Modified Mackenzie Sound Equation, gaussian process and Golden Ratio Optimizations Based CVOA Algorithm Routing in UASN

\subsection{Modified Mackenzie Equation (MME)}

Mackenzie Equation is as in Equation 1 and measures c=acoustic velocity base on the T, S and D.

$$
\begin{aligned}
\mathrm{C}(\mathrm{D}, \mathrm{S}, \mathrm{T})= & 1448.96+4.591 \mathrm{~T}-5.304 \times 10^{-2} \mathrm{~T}^{2}+2.374 \times 10^{-4} \mathrm{~T}^{3}+1.340(\mathrm{~S}-35)+1.630 \times \\
& 10^{-2} \mathrm{D}+1.675 \times 10^{-7} \mathrm{D}^{2}-1.025 \times 10^{-2} \mathrm{~T}(\mathrm{~S}-35)-7.139 \times 10^{-13} \mathrm{TD}^{3}
\end{aligned}
$$

where $\mathrm{T}=$ temperature in degrees Celsius, $\mathrm{S}=$ salinity in parts per thousand, $\mathrm{D}=$ depth in meters, above equation is modified as in Equation 2

$$
\mathrm{C}\left(\mathrm{D}, \mathrm{S}, \mathrm{T}, \mathrm{DCO}_{2}\right)=1448.96+4.591 \mathrm{~T}-5.304 \times 10^{-2} \mathrm{~T}^{2}+2.374 \times 10^{-4} \mathrm{~T}^{3}+1.340(\mathrm{~S}-35)+1.630 \times
$$

where $\mathrm{f}(\mathrm{DCO} 2)=$ function of dissolved $\mathrm{CO} 2$.

\subsection{Weather Observations Through Ambient Noise (WOTAN)}

In each node, environmental parameters such as temperature, depth, salinity, dissolved gases, pressure, and sound velocity, is measured, and then rotational, and divergent wind is measured through WOTAN. WOTAN measures wind speed based on underwater ambient noise. Underwater ambient noise produces due to excitations on ocean/sea surface and change in weather conditions. WOTAN measures rotational, divergent wind stress estimated through the linear relationship between sound velocity pressure and surface wind speed is exploited and measures wind speed, which is given as:

$$
\mathrm{sv}=\mathrm{O}+(\mathrm{m} * \mathrm{sws})
$$

where $\mathrm{sv}-$ sound velocity pressure measured by $\mu \mathrm{Pa}$

$\mathrm{o}-$ offset, measured by $\mu \mathrm{Pa}$ 
$\mathrm{m}$ - slope, measured by $\mu \mathrm{Pa} \mathrm{m}-1 \mathrm{~s}$

sws - surface wind speed, measured by ms-1

The sound pressure level $3 \mathrm{kHz} 1 / 3$-octave band shows an active response to the speed of wind which varies from 2 to $21.5 \mathrm{~m} / \mathrm{s} .21 .5 \mathrm{~m} / \mathrm{s}$ is characterized as a strong wind level.

\subsection{Gaussian Process Regression (GPR)}

Every node has a dataset of all parameters such as temperature, depth, salinity, dissolved gases, pressure, sound velocity (MME), is measured rotational, and divergent wind (WOTAN) then process with GPR for propagation delay estimation. Fig. 2 depicts the relationship between depth and acoustic speed. Fig. 3 shows the relationship between temperature and acoustic speed. Fig. 4 shows the relationship between salinity and acoustic speed.

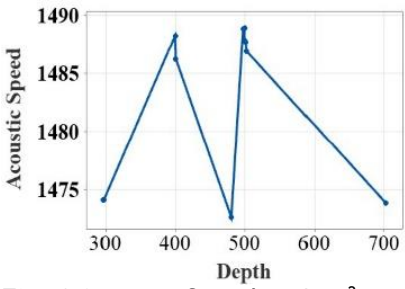

Fig. 1 Acoustic Speed $\left(\mathrm{m} / \mathrm{sec}^{2}\right)$ Vs Depth $(\mathrm{m})$

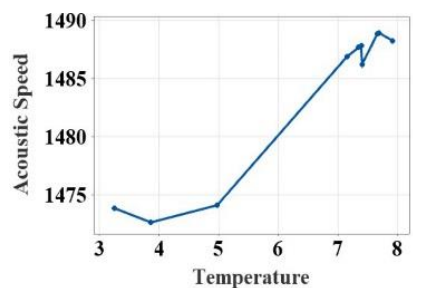

Fig. 2 Acoustic Speed $\left(\mathrm{m} / \mathrm{sec}^{2}\right)$ Vs Temperature $(C)$

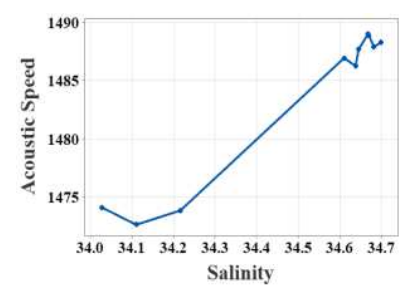

Fig. 3 Acoustic Speed $\left(\mathrm{m} / \mathrm{sec}^{2}\right)$ Vs Salinity (PSU)

Gaussian Process Regression (GPR) is a nonparametric regression model and uses the Bayesian concept. GPR provides a better prediction for small datasets and provides the prediction's uncertainty estimation. GPR distribution infers from function. Gaussian processes are treated as a prior to build predictive posterior distribution given by Equation 4 .

$$
\mathrm{f}(\mathrm{x}) \sim \mathrm{GP}\left(\mathrm{m}(\mathrm{x}), \mathrm{k}\left(\mathrm{x}, \mathrm{x}^{\prime}\right)\right)
$$

where $\mathrm{m}(\mathrm{x})$ is the mean function and $\mathrm{k}\left(\mathrm{x}, \mathrm{x}^{\prime}\right)$ is a covariance function. The Squared exponential kernel or covariance function models smooth functions. The Squared exponential kernel provides infinitely many derivatives from prior functions, which is given by Equation 5.

$$
\mathrm{k}\left(\mathrm{x}, \mathrm{x}^{\prime}\right)=\sigma^{2} \exp \left(-\frac{\left(\mathrm{x}-\mathrm{x}^{\prime}\right)^{2}}{21^{2}}\right)
$$

where 1 smoothens the function and $\sigma 2$ checks the variation of function vertically.

The Equation 6 shows the relationship of acoustic velocity with

$$
\mathrm{C}=1441.7913+0.0174 \times \mathrm{D}+4.2254 \times \mathrm{T}+0.1668 \times \mathrm{S}
$$

where $\mathrm{c}$ is acoustic speed, $\mathrm{D}$ is depth, $\mathrm{T}$ is temperature and $\mathrm{S}$ is salinity.

Table 2 shows the estimate and standard error for the regression model for acoustic speed and its predictors depth, temperature, and salinity.

Table 2 Estimates and standard error for prediction of acoustic speed

\begin{tabular}{llrrrr}
\hline Predictor & Coefficient & \multicolumn{1}{c}{ Estimate } & \multicolumn{1}{c}{$\begin{array}{c}\text { Standard } \\
\text { Error }\end{array}$} & \multicolumn{1}{c}{ tt-statistic } & pp-value \\
\hline Constant & $\beta 0 \beta 0$ & 1441.7913 & 3.4337 & 419.896 & 0 \\
DD & $\beta 1 \beta 1$ & 0.0174 & 0.0001 & 333.0335 & 0 \\
TT & $\beta 2 \beta 2$ & 4.2254 & 0.0328 & 129.0123 & 0 \\
SS & $\beta 3 \beta 3$ & 0.1668 & 0.1034 & 1.6121 & 0.11 \\
\hline
\end{tabular}


Table 3 depicts the overall fit for the above discussed parameters

Table 3 Estimates and standard error for prediction of acoustic speed

\begin{tabular}{lll}
\hline Residual Standard Error & Overall FF-statistic & Overall pp-value \\
\hline $\begin{array}{l}0.4319 \text { on } 103 \text { degrees of } \\
\text { freedom. }\end{array}$ & $\begin{array}{l}108413.0879 \text { on } 3 \text { and } \\
103 \text { degrees of freedom. }\end{array}$ & 0 \\
\hline
\end{tabular}

Fig. 5 shows histogram of residuals.

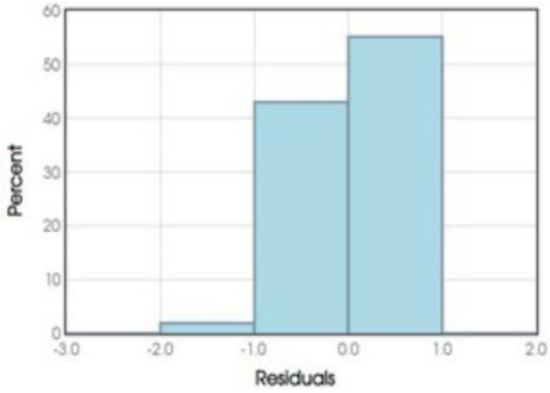

Fig. 4 Histogram of residuals

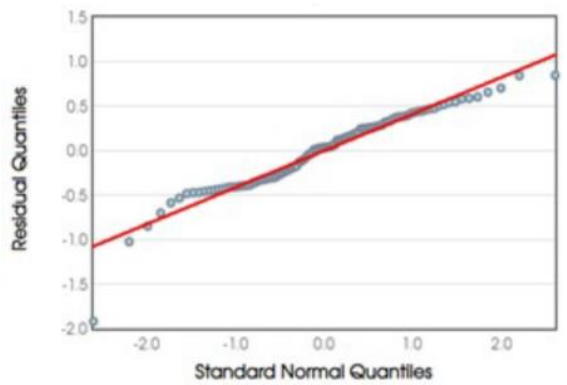

Fig. 5 Normal Probability of Residuals

Fig. 6 shows normal probability of residuals. GPR predicts the nodes with low propagation delay.

\subsection{Golden Ration Optimization Method (GROM)}

GROM is a parameter-less meta-heuristic optimization method, which is inspired by the golden ratio or golden mean found in the growth of plants and animals [32]. Fibonacci, a renowned mathematician, formulated this ratio by a series of numbers generated from the sum of the previous two numbers. Except for the first two numbers, all other numbers can be generated. The ratio of any two consecutive numbers is approximately the same i.e., 1.618, and represented as $\Phi$. This kind of pattern is common in the arrangement of petals in flowers, leaves, pinecones, shells, hurricanes. Any kth number $\mathrm{x}$, such that $\mathrm{k}>=3$, in the Fibonacci series is generated by using this golden ratio, 1.618, which is given by Equation 7 :

$$
\mathrm{X}_{\mathrm{k}}=\frac{\Phi^{\mathrm{k}}-(1-\Phi)^{\mathrm{k}}}{\sqrt{5}}
$$

In GROM, the fitness mean of the population is computed and compared with the worst solution. To increase the speed of convergence, worst solution is replaced by the mean solution, if the fitness of the mean solution is better than the worst solution. A random vector from the population is selected for each vector of population. The fitness of these two vectors is compared with the mean solution. The solutions are arranged as $\mathrm{x}_{\text {best }}, \mathrm{x}_{\text {medium }}$ and $\mathrm{x}_{\text {worst }}$. To identify the direction of movement of vectors and amount of movement is given by Equation 8

$$
\overrightarrow{\mathrm{x}_{\mathrm{t}}}=\underset{\mathrm{x}_{\text {medium }}}{\longrightarrow}-\underset{\mathrm{x}_{\mathrm{worst}}}{\longrightarrow}
$$

The fitness function is given by Equation 9

$$
\mathrm{F}_{\mathrm{t}}=\mathrm{GF} \times \frac{\Phi^{\mathrm{T}}-(1-\Phi)^{\mathrm{T}}}{\sqrt{5}}
$$


where $\mathrm{GF}=1.618$ and $\mathrm{T}=\mathrm{t} / \mathrm{tmax}$

The new vector is computed as in Equation 10

$$
\mathrm{x}_{\text {new }}=\left(1-\mathrm{F}_{\mathrm{t}}\right) \times \mathrm{x}_{\text {best }}+\text { rand } \times \mathrm{F}_{\mathrm{t}} \times \mathrm{x}_{\mathrm{t}}
$$

Based on boundary conditions, xnew replaces the original solution, if the computed new solution is better than the original. The second phase attempts to move for solutions closer to the best solution based on Equation 11,

where, $1 / \mathrm{GF}=0.618$

$$
\mathrm{x}_{\text {new }}=\mathrm{x}_{\text {old }}+\text { rand } \times\left(\frac{1}{\mathrm{GF}}\right) \times\left(\mathrm{x}_{\text {best }}-\mathrm{x}_{\text {worst }}\right)
$$

The boundary conditions are checked and the old vector replaces with newly compute values if the new vector is better than the old one.

\subsection{Corona Virus Optimization Algorithm (CVOA)}

In recent times, many metaheuristic algorithms inspired by nature is proposed and finds the optimal solution for different problems. COVID-19, Corona Virus Optimization algorithm (CVOA) [33] is one such metaheuristic algorithm, which simulates Coronavirus spread and infects healthy individuals. Patient zero (PZ) is the first infected individual, spreads the virus to other healthy individuals. The infected individual may either die or spread infection. Initially, the infection spreads exponentially and later decrease due to recovery or deaths in the population. The disease propagates from PZ. The initial population is generated with prime infected individual PZ. PZ infects other individuals in the population. Each infected individual die either based on probability P-_DIE or spread infection. Based on probability P_SUPERSPREADER, spreaders are differentiated into Ordinary spreaders and Superspreaders. The ordinary spreader spreads infection based on SPREADING_RATE, whereas super-spreader infects population-based on SUPERSPREADING_RATE. CVOA assumes some of the infected individuals travel for diversification. Each infected individual travel with probability P_TRAVEL. Based on this probability, the infected individual either travels with TRAVELER_RATE or if not travels in ORDINARY_RATE. An infected individual can be both a super-spreader or a traveler.

In each generation, three types of population namely deaths, recovered, the new infected population are observed and updated. The dead individuals are added to this population and cannot be reused. At the start of every iteration, all infected populations are added to recover the population. The individual recovered can be reinfected with probability P_REINFECTION. When an individual is infected, he/she might be isolated to follow social distancing norms. Such isolated individuals may be added to the recovered population with probability P_ISOLATION. All infected population in each iteration is added to the new infected population. The stop criterion is a pre-set number of iterations. In initial iterations, infections will grow exponentially, along with it grows recovered population and dead population. The below algorithm explains the use of the CVOA algorithm in UASN. In this paper, Sedimentation drift causes rotational, divergent wind stress and considered to be the virus that affects the nodes in UASN. The CVOA algorithm is as below.

1. Input the required parameters

2. time $\leftarrow 0$

3. infectedNodes $\leftarrow \mathrm{x}$

4. nextHop $\leftarrow \mathrm{x}$

5. while (time < PANDEMIC_DUR and sizeof(infectednodes) $>0$ )

6. dead $\leftarrow$ noBattery(infectednodes)

7.

for each i in infectednodes
i. $\quad$ aux $\leftarrow$ infect $(\mathrm{i}$, recovered, dead)
ii. $\quad$ if notnull(aux) then

newInfectedNodes $\leftarrow$ aux




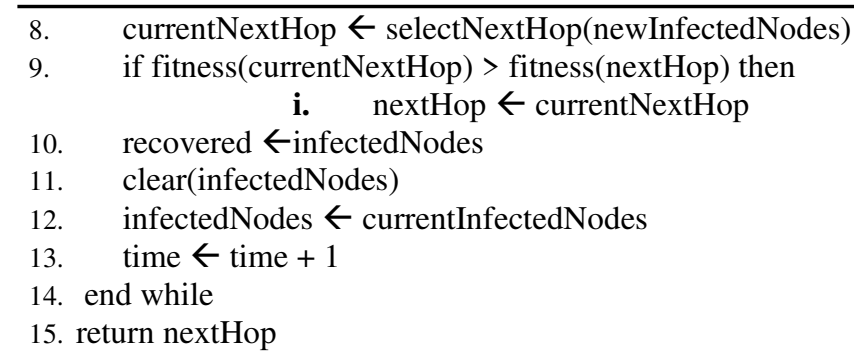

\section{Results and Discussion}

Fig 7 shows the system model. The system is initialized to contain 50 nodes. For every 30 minutes node parameters such as temperature, pressure, salinity, dissolved gases, rotational and divergent wind stress estimated using Mackenzie equation and WOTAN estimation, such observed data stored in node database. The node with an acoustic velocity greater than 1527 $\mathrm{m} / \mathrm{sec}$ is selected for the next step.

In this paper, Gaussian Process calculates propagation delay in each node based on the database in each node, and the nodes with less propagation delay are only considered for routing through the CVOA algorithm as shown in Fig 8. However, the performance of the Gaussian Process in the prediction of propagation delay, in each node, is replaced with the Golden Ratio algorithm and then the CVOA algorithm is applied. The selection of nodes, through the Gaussian Process algorithm and Golden ratio algorithm, plays a vital role for energy-aware routing in the network through appropriate node selections. The CVOA routing algorithm applied in two scenarios such as

(i) Gaussian Process and CVOA algorithm

(ii) Golden Ratio and CVOA algorithm 


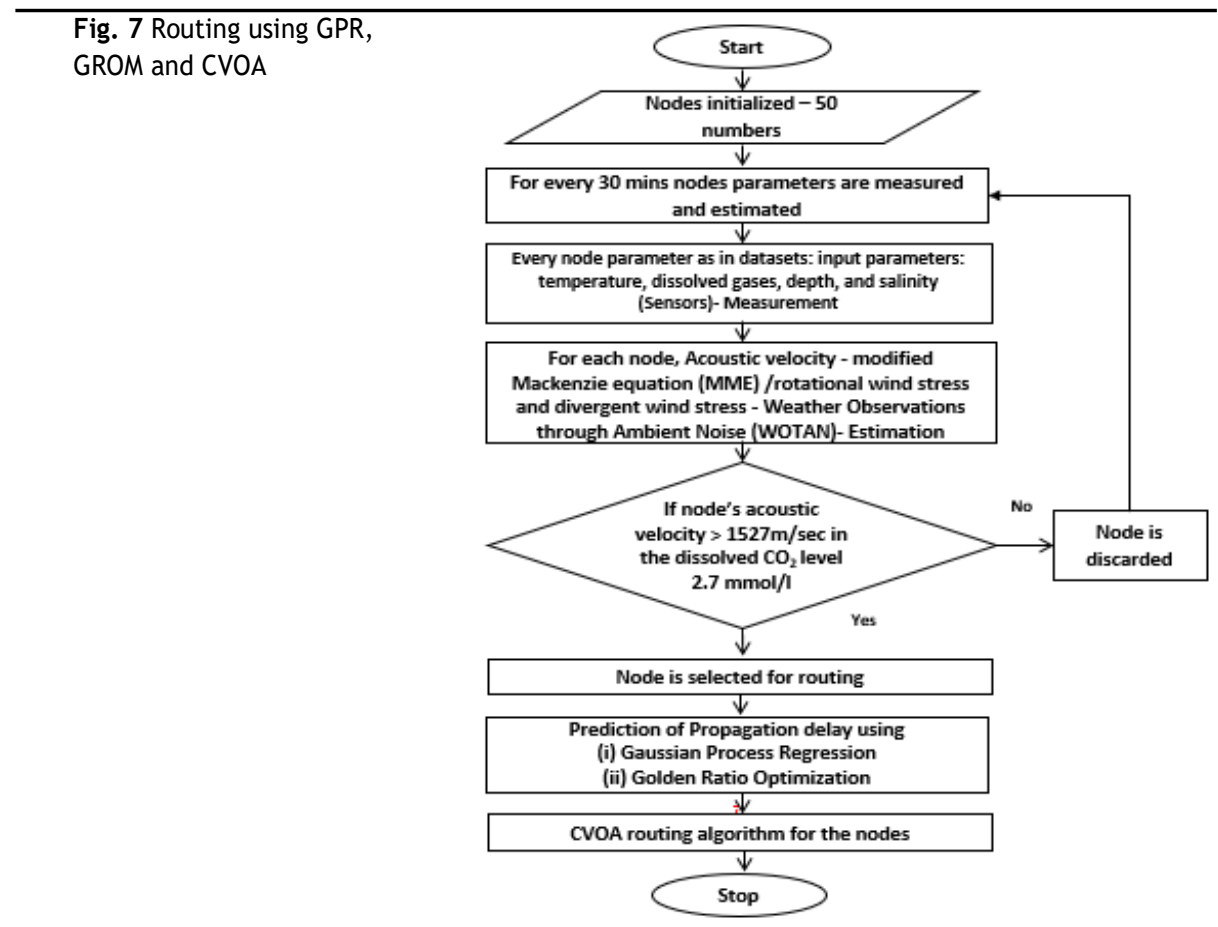

\subsection{GPR AND CVOA based Routing}

CVOA finds the best route after selecting the nodes with less propagation delay. The source is considered as PZ. Based on the acoustic velocity of each neighboring nodes' region, the node is added to the UASN network.

Sedimentation drift causes rotational, divergent wind stress and considered to be the virus that affects nodes in UASN. Each node is considered either as an ordinary spreader or a super-spreader considering its battery level. The node with a high battery is considered to be high immunity and considered to be an ordinary-spreader. The node with less battery level is a super-spreader as sedimentation drift drains the sensor battery. The node that has no battery left is considered as a dead node. A node can be with good acoustic velocity region and good battery level and away from formed UASN, the node is considered as a node maintaining social distancing and will not be part of the network. Fig. 8 shows the routing of CVOA. 


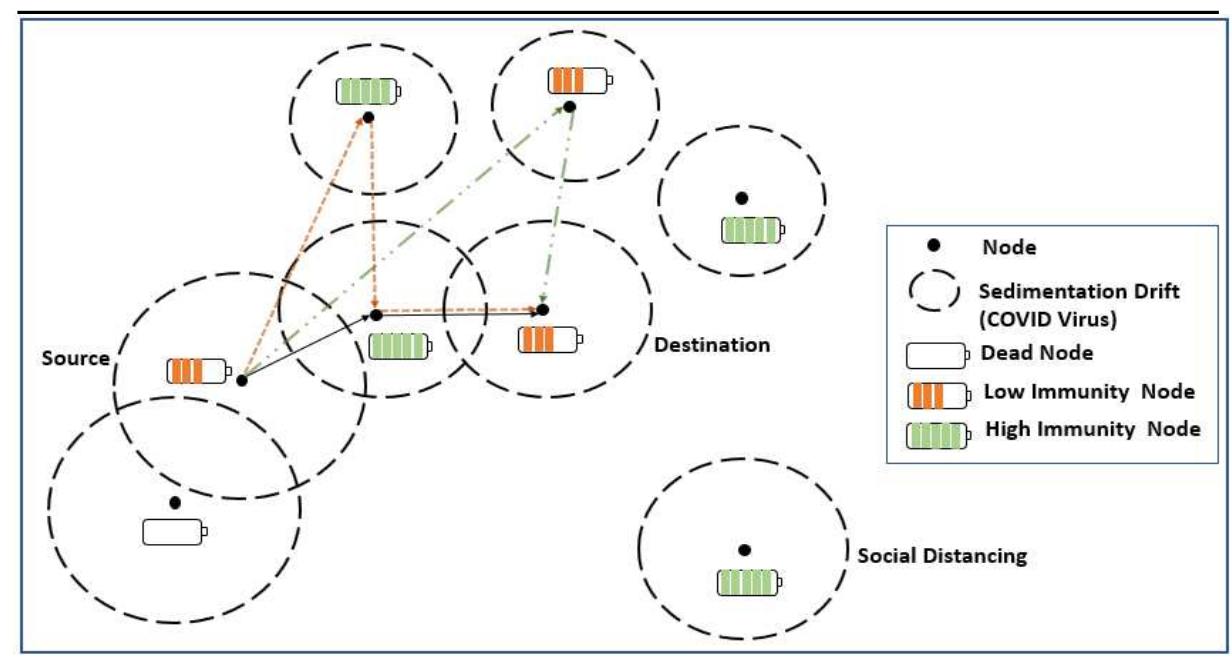

Fig. 8 CVOA in UASN for routing

The following is the GPR and CVOA Algorithm:

1. initialize the required parameters

2. $\mathrm{x} \leftarrow$ source

3. while ( $\mathrm{x}$ is not Destination)

4. append $\mathrm{x}$ to the route

5. for each neighbouring node of $\mathrm{x}$, compute the acoustic velocity in its region using modified Mackenzie equation and WOTAN estimation

6. Calculate the average $\mathrm{AV}_{\mathrm{avg}}$ of all the nodes computed in the previous step.

7. for each neighbouring node of $\mathrm{x}$,

a. If node's acoustic velocity $>=A V_{\text {avg }}$, then append this node in the list $\mathrm{AV}$

8. for each node in AV, predict the propagation delay using GPR

9. Calculate the average propagation delay, $\mathrm{PD}_{\text {avg }}$ of all the nodes computed in the previous step.

10. for each node in AV

a. if the node's propagation delay $<=\mathrm{PD}_{\mathrm{avg}}$, then append this node in the list PD

11. apply CVOA to find the next hop with input population as PD and $\mathrm{x}$ as source $\mathrm{x} \leftarrow$ nextHop

12. end while

13. return route

\subsection{GROM AND CVOA based Routing}

The following is the GROM and CVOA Algorithm:

1. initialize the required parameters

2. $x \leftarrow$ source

3. while ( $\mathrm{x}$ is not Destination)

4. append $\mathrm{x}$ to the route

5. for each neighboring node of $\mathrm{x}$, compute the acoustic velocity in its region using modified Mackenzie equation and WOTAN estimation

6. Calculate the average $\mathrm{AV}_{\mathrm{avg}}$ of all the nodes computed in the previous step.

7. for each neighboring node of $\mathrm{x}$, 
a. If node's acoustic velocity $>=A V_{\text {avg }}$, then append this node in the list $\mathrm{AV}$

8. for each node in AV, predict the propagation delay using GROM

9. Calculate the average propagation delay, $\mathrm{PD}_{\text {avg }}$ of all the nodes computed in the previous step.

10. for each node in AV

a. if the node's propagation delay $<=\mathrm{PD}_{\mathrm{avg}}$, then append this node in the list $\mathrm{PD}$

11. apply CVOA to find the next hop with input population as PD and $\mathrm{x}$ as source $\mathrm{x} \leftarrow$ nextHop

12. end while

13. return route

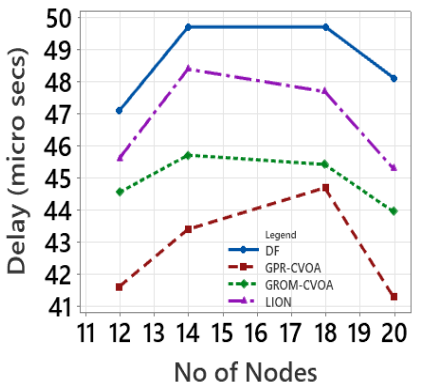

Fig. 9 Delay Vs Number of Nodes

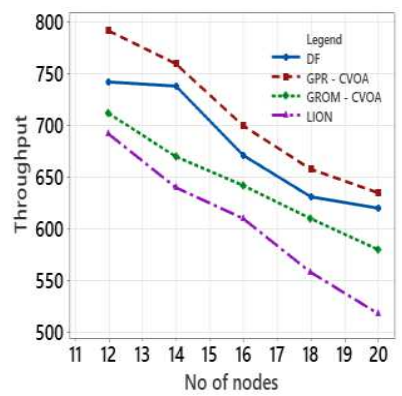

Fig. 12 Throughput Vs Number of Nodes

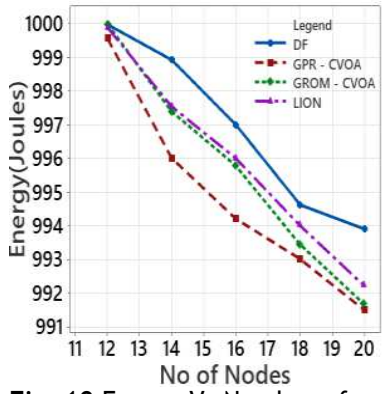

Fig. 10 Energy Vs Number of Nodes

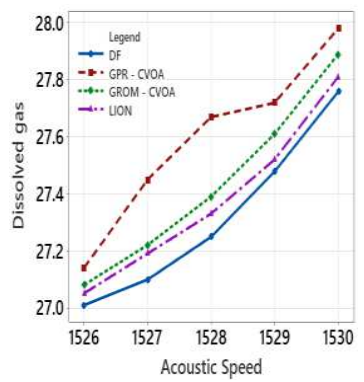

Fig. 13 Dissolved gas Vs Acoustic Speed

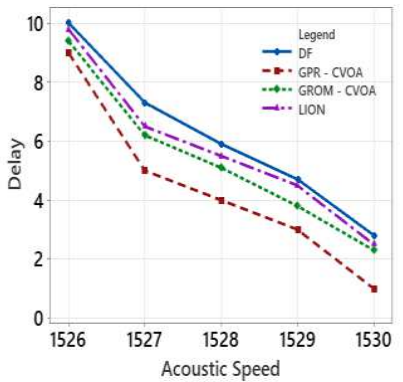

Fig. 11 Acoustic Speed Vs Delay

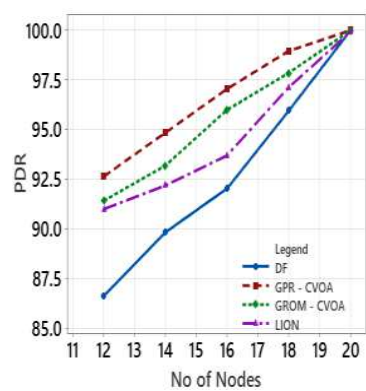

Fig. 14 PDR Vs Number of Nodes 


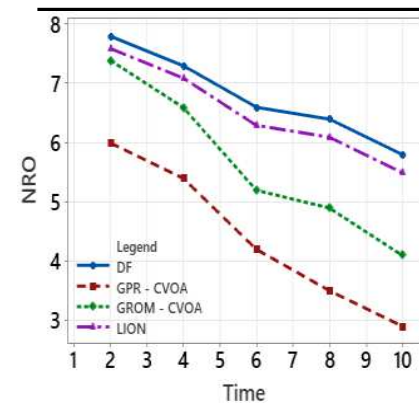

Fig. 15 NRO Vs Time (millisecs)

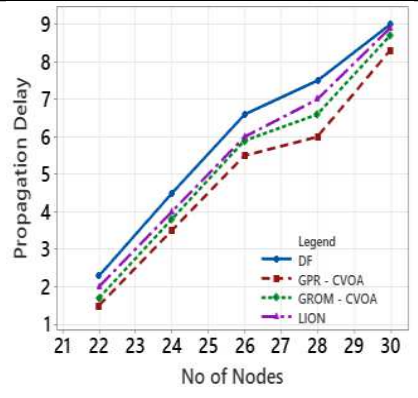

Fig. 16 Propagation Delay vs Number of Nodes in the presence of Rotational Wind

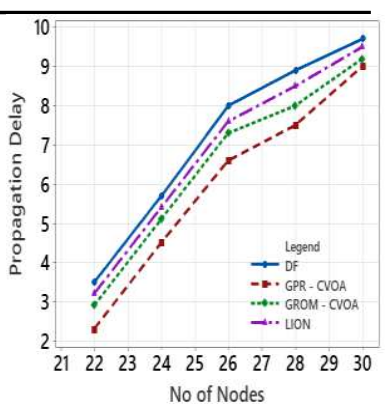

Fig. 17 Propagation Delay vs Number of Nodes in the presence of Divergent Wind

The performance of GPR-CVOA and GROM-CVOA is compared with the Direct Forwarding (DF) algorithm and LION algorithm [10]. Fig. 9 illustrates the effects of the number of nodes on delay. GPR-CVOA is performing better when compared to DF, GROMCVOA, and LION. Fig. 10 analysis energy consumption when the no of nodes varied. It is observed that energy consumption reduces as the number of nodes in the network increases. The energy consumption of GROM-CVOA is close to that of the LION protocol's energy consumption. Energy consumption is less in GPR-CVOA, GROM-CVOA than DF and LION.

Fig. 11 expresses the relationship between acoustic speed and delay and they are inversely proportional to each other. GPR-CVOA outperforms other algorithms and has minimal delay than other protocols like GROM-CVOA, DF, and LION. Fig. 12 illustrates throughput versus the number of nodes. The graph shows that GPR-CVOA has more throughput when compared to other protocols. Fig. 13 illustrates the effect of dissolved gases vs acoustic speed

Fig. 14 shows the effects of the number of nodes on PDR is analyzed. Both GPR-CVOA and GROM-CVOA protocols has better PDR than LION and DF. Both GPR-CVOA and GROM-CVOA protocols has a good initial PDR than DF as water-column properties, dissolved gases, wind patterns are accounted for while calculating the route for packet transmission. Fig. 15 illustrates Normalized Routing Overhead (NRO) versus time. NRO is less in GPR-CVOA and GROM-CVOA than DF and LION.

Fig. 16 shows that in presence of rotational wind, propagation delay increases with an increase in the number of nodes. The GPR-CVOA and GROM-CVOA protocols perform better than DF and LION. Fig. 17 illustrates the propagation delay versus the number of nodes in presence of divergent wind. GPR-CVOA performs well than the GROM-CVOA protocol but both are better than the other two protocols under study.

Performance of GPR-CVOA and GROM-CVOA shows that under changing wind patterns and dissolved gases, protocols are robust and prove to be working better than DF and LION.

\section{Conclusion}

In UASN, routing and propagation delay is affected because of temperature, salinity, depth, dissolved gases, sound velocity, divergent and rotational wind. Modified Mackenzie Sound equation is proposed and calculate sound velocity in each node for energy-efficient routing. Golden Ratio Optimization Method (GROM) and Gaussian Process Regression (GPR) predict propagation delay of each node based on dissolved gases, rotational and divergent wind stress. Predicted values from GPR and GROM lead to node selection and among selected nodes Corona Virus Optimization Algorithm (CVOA) routing is performed. The 
proposed GPR-CVOA and GROM-CVOA algorithm solves the problem of propagation delay and consumes less energy in nodes based on appropriate tolerant delays in transmitting packets among nodes during high rotational and divergent winds. To reduce, number of sensors in node and improve the energy efficiency of the node through Mackenzie equationempirical method. Mackenzie equation replaces sensors such as wind, wind direction, sedimentation drift, Doppler Effect, and geometric spread through the sound profile calculation obtaining from the Mackenzie equation. In this paper, a modified Mackenzie equation (MME) is proposed for the estimation of sound velocity through dissolved gases. In UASN, routing based on water column variations especially for divergent and rotational wind environments are designed. This paper predicts ocean parameters like dissolved gases through MME, depth, salinity, and temperature through sensors, rotational and divergent wind stress through prediction algorithms such as GROM and GPR. MME calculates the sound velocity of the node region, which includes dissolved gases. MME helps to calculate the divergent and rotational wind through sound profile with Weather Observations through Ambient Noise (WOTAN). CVOA algorithm is applied for routing in UASN to avoid propagation delay due to dissolved gases, sedimentation drift, and water column variations. After predicting each node's environmental conditions, nodes are selected for CVOA Algorithms and proposed algorithms perform better than existing water column variation algorithms. The prediction algorithm can be applied with deep learning algorithms for other water column variation, which consumes more power such as sand drift and turbulence.

\section{Declarations}

\section{Funding}

Not Applicable

\section{Conflicts of interest}

The authors declare that there is no conflict of interest regarding the publication of this paper.

\section{Availability of data and material}

The data used to support the findings of this study are available from the corresponding author upon request.

\section{Code availability}

NS2 code in aquasim

https://drive.google.com/drive/folders/1Hh22hNLSCr_FlZJZUvjM2Pv0n_wsdY6?usp=shari $\underline{\mathrm{ng}}$

\section{References}

1. Su, Y., Guo, L., Jin, Z., \& Fu, X. (2020). A Voronoi-Based Optimized Depth Adjustment Deployment Scheme for Underwater Acoustic Sensor Networks. IEEE Sensors Journal, 20(22), 13849-13860.

2. Jin, Z., Zhao, Q., \& Luo, Y. (2020). Routing void prediction and repairing in AUV-assisted underwater acoustic sensor networks. IEEE Access, 8, 54200-54212.

3. Su, Y., Zhang, L., Li, Y., \& Yao, X. (2020). A Glider-Assist Routing Protocol for Underwater Acoustic Networks with Trajectory Prediction Methods. IEEE Access, 8, 154560-154572.

4. Guan, Q., Ji, F., Liu, Y., Yu, H., \& Chen, W. (2019). Distance-vector-based opportunistic routing for underwater acoustic sensor networks. IEEE Internet of Things Journal, 6(2), 3831-3839.

5. Su, Y., Fan, R., Fu, X., \& Jin, Z. (2019). DQELR: An adaptive deep Q-network-based energy-and latency-aware routing protocol design for underwater acoustic sensor networks. IEEE Access, 7, 9091-9104.

6. Jin, Z., Zhao, Q., \& Su, Y. (2019). RCAR: A reinforcement-learning-based routing protocol for 
congestion-avoided underwater acoustic sensor networks. IEEE Sensors Journal, 19(22), 1088110891.

7. Lin, C., Han, G., Guizani, M., Bi, Y., \& Du, J. (2019). A scheme for delay-sensitive spatiotemporal routing in SDN-enabled underwater acoustic sensor networks. IEEE Transactions on Vehicular Technology, 68(9), 9280-9292.

8. Jin, Z., Ji, Z., \& Su, Y. (2018). An evidence theory based opportunistic routing protocol for underwater acoustic sensor networks. IEEE Access, 6, 71038-71047.

9. NR, S., \& Samuel, B. E. (2020). Improving Packet Delivery Performance in Water Column Variations through LOCAN in Underwater Acoustic Sensor Network. Journal of Sensors, 2020.

10. Clarke, J. S., Achterberg, E. P., Connelly, D. P., Schuster, U., \& Mowlem, M. (2017). Developments in marine pCO2 measurement technology; towards sustained in situ observations. TrAC Trends in Analytical Chemistry, 88, 53-61.

11. Wang, Q., Li, J., Qi, Q., Zhou, P., \& Wu, D. O. (2020). A Game-Theoretic Routing Protocol for 3-D Underwater Acoustic Sensor Networks. IEEE Internet of Things Journal, 7(10), 9846-9857.

12. Su, Y., Guo, L., Jin, Z., \& Fu, X. (2020). A Mobile-beacon Based Iterative Localization Mechanism in Large-scale Underwater Acoustic Sensor Networks. IEEE Internet of Things Journal.

13. Wang, L., Yang, Y., \& Liu, X. (2020). A Direct Position Determination Approach for Underwater Acoustic Sensor Networks. IEEE Transactions on Vehicular Technology, 69(11), 13033-13044.

14. Han, G., Du, J., Lin, C., Wu, H., \& Guizani, M. (2019). An energy-balanced trust cloud migration scheme for underwater acoustic sensor networks. IEEE Transactions on Wireless Communications, 19(3), 1636-1649.

15. Zhang, W., Han, G., Wang, X., Guizani, M., Fan, K., \& Shu, L. (2020). A node location algorithm based on node movement prediction in underwater acoustic sensor networks. IEEE Transactions on Vehicular Technology, 69(3), 3166-3178.

16. Han, G., He, Y., Jiang, J., Wang, N., Guizani, M., \& Ansere, J. A. (2019). A synergetic trust model based on SVM in underwater acoustic sensor networks. IEEE Transactions on Vehicular Technology, 68(11), 11239-11247.

17. Zhao, R., Li, N., Dobre, O. A., \& Shen, X. (2020). CITP: collision and interruption tolerant protocol for underwater acoustic sensor networks. IEEE Communications Letters, 24(6), 1328-1332.

18. Yan, J., Guo, D., Luo, X., \& Guan, X. (2020). AUV-Aided localization for underwater acoustic sensor networks with current field estimation. IEEE Transactions on Vehicular Technology, 69(8), 88558870 .

19. Zhuo, X., Liu, M., Wei, Y., Yu, G., Qu, F., \& Sun, R. (2020). AUV-aided energy-efficient data collection in underwater acoustic sensor networks. IEEE Internet of Things Journal, 7(10), 1001010022.

20. Li, X., Zhou, Y., Yan, L., Zhao, H., Yan, X., \& Luo, X. (2019). Optimal node selection for hybrid attack in underwater acoustic sensor networks: A virtual expert-guided bandit algorithm. IEEE Sensors Journal, 20(3), 1679-1687.

21. Bouabdallah, F., Boutaba, R., \& Mehaoua, A. (2018). Collision avoidance energy efficient multichannel MAC protocol for underwater acoustic sensor networks. IEEE Transactions on Mobile Computing, 18(10), 2298-2314.

22. Zhou, Y., Yang, H., Hu, Y. H., \& Kung, S. Y. (2019). Cross-layer network lifetime maximization in underwater wireless sensor networks. IEEE Systems Journal, 14(1), 220-231.

23. Jiang, W., Tong, F., Zheng, S., \& Cao, X. (2020). Estimation of Underwater Acoustic Channel With Hybrid Sparsity via Static-Dynamic Discriminative Compressed Sensing. IEEE Sensors Journal, 20(23), 14548-14558.

24. Du, J., Han, G., Lin, C., \& Martinez-Garcia, M. (2020). ITrust: An Anomaly-resilient Trust Model Based on Isolation Forest for Underwater Acoustic Sensor Networks. IEEE Transactions on Mobile Computing.

25. Kim, Y., Erol-Kantarci, M., Noh, Y., \& Kim, K. (2020). Range-Free Localization with a Mobile Beacon via Motion Compensation in Underwater Sensor Networks. IEEE Wireless Communications Letters.

26. Morozs, N., Mitchell, P. D., \& Diamant, R. (2020). Scalable Adaptive Networking for the Internet of Underwater Things. IEEE Internet of Things Journal, 7(10), 10023-10037.

27. Han, G., Shen, S., Wang, H., Jiang, J., \& Guizani, M. (2019). Prediction-based delay optimization data collection algorithm for underwater acoustic sensor networks. IEEE Transactions on Vehicular Technology, 68(7), 6926-6936.

28. Rahmati, M., \& Pompili, D. (2018). Probabilistic spatially-divided multiple access in underwater acoustic sparse networks. IEEE Transactions on Mobile Computing, 19(2), 405-418.

29. Guida, R., Demirors, E., Dave, N., \& Melodia, T. (2020). Underwater Ultrasonic Wireless Power Transfer: A Battery-less Platform for the Internet of Underwater Things. IEEE Transactions on Mobile Computing.

30. Song, Y. (2020). Underwater acoustic sensor networks with cost efficiency for internet of underwater 
things. IEEE Transactions on Industrial Electronics, 68(2), 1707-1716.

31. Cauchy, P., Heywood, K. J., Merchant, N. D., Queste, B. Y., \& Testor, P. (2018). Wind speed measured from underwater gliders using passive acoustics. Journal of Atmospheric and Oceanic Technology, 35(12), 2305-2321.

32. Nematollahi, A. F., Rahiminejad, A., \& Vahidi, B. (2020). A novel meta-heuristic optimization method based on golden ratio in nature. Soft Computing, 24(2), 1117-1151.

33. Martínez-Álvarez, F., Asencio-Cortés, G., Torres, J. F., Gutiérrez-Avilés, D., Melgar-García, L., Pérez-Chacón, R., ... \& Troncoso, A. (2020). Coronavirus Optimization Algorithm: A bioinspired metaheuristic based on the COVID-19 propagation model. Big Data, 8(4), 308-322. 


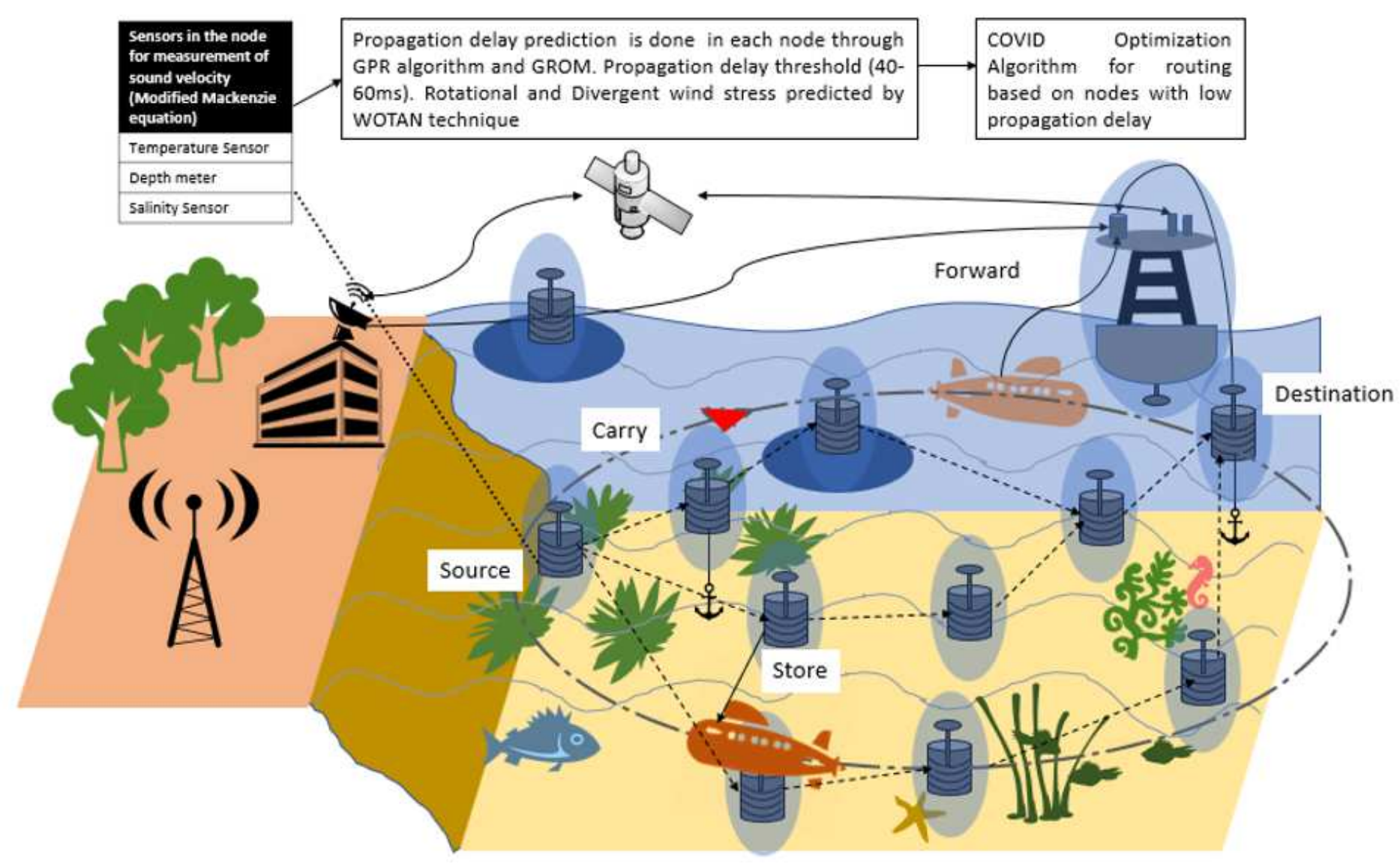

\section{Figure 1}

Modified Mackenzie Sound Equation, gaussian process and Golden Ratio Optimizations Based CVOA Algorithm Routing in UASN

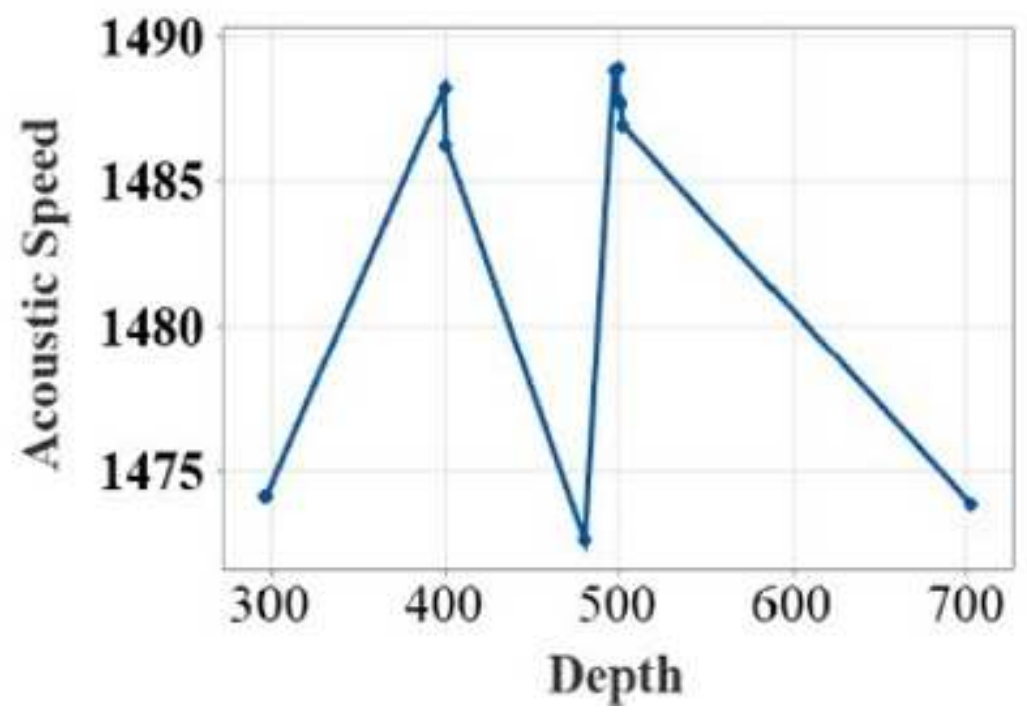

Figure 2 
Acoustic Speed (m/sec2) Vs Depth (m)

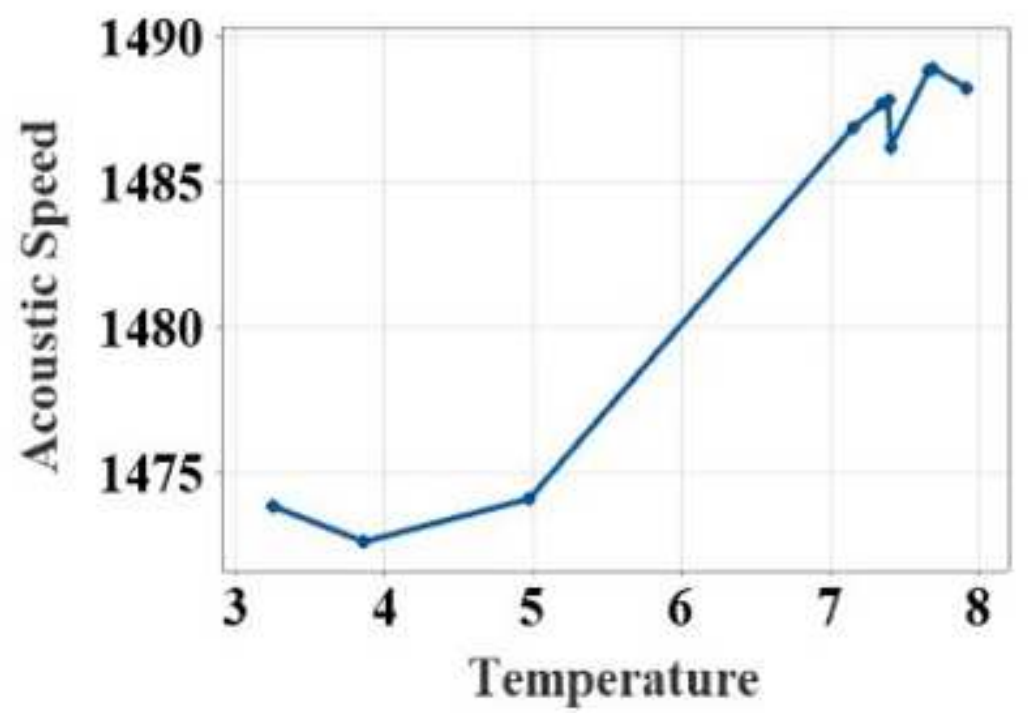

Figure 3

Acoustic Speed (m/sec2) Vs Temperature (C)

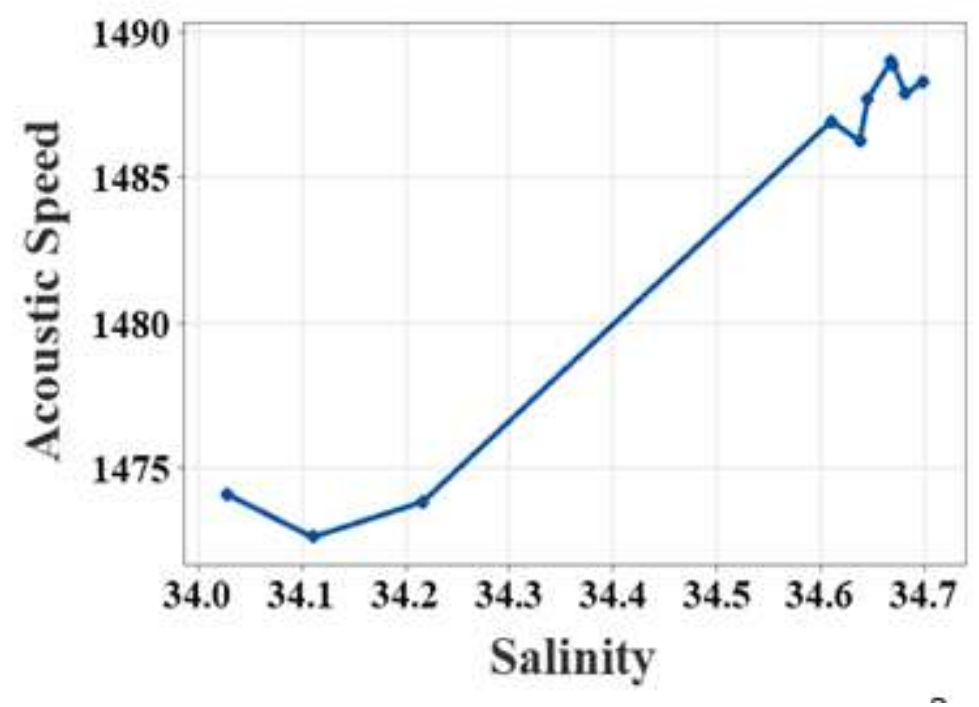

Figure 4

Acoustic Speed (m/sec2) Vs Salinity (PSU) 


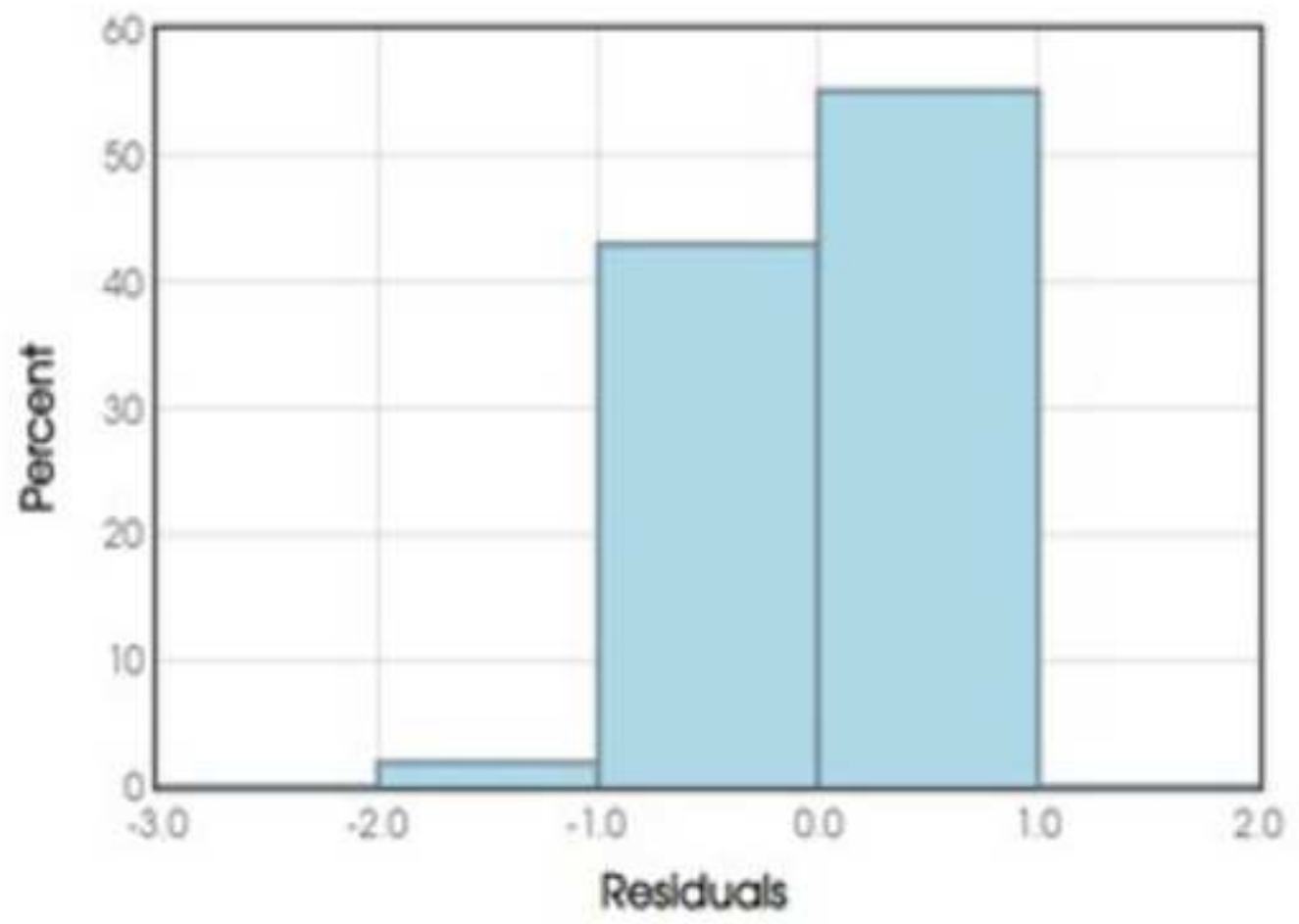

Figure 5

Histogram of residuals

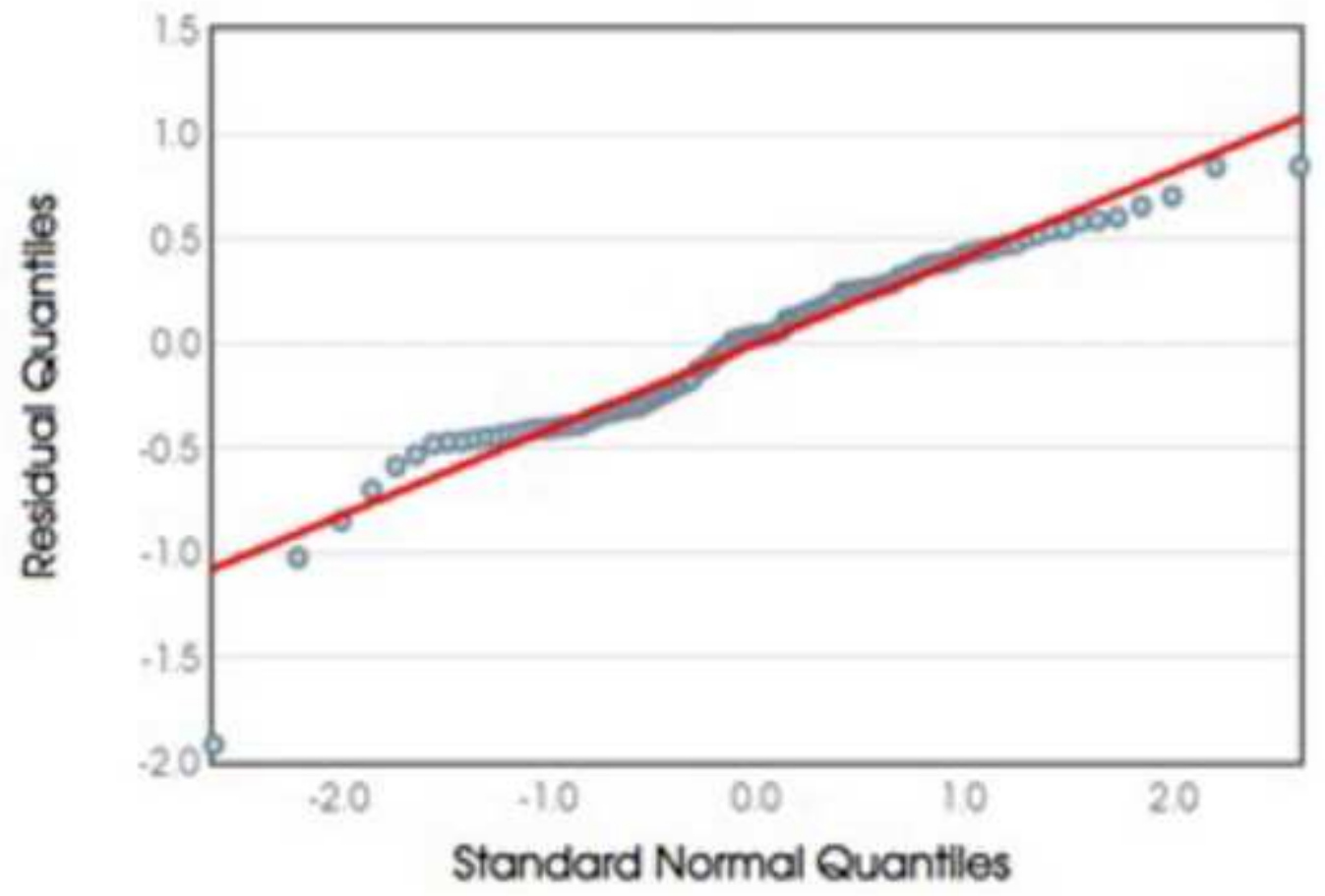

Figure 6

Normal Probability of Residuals 


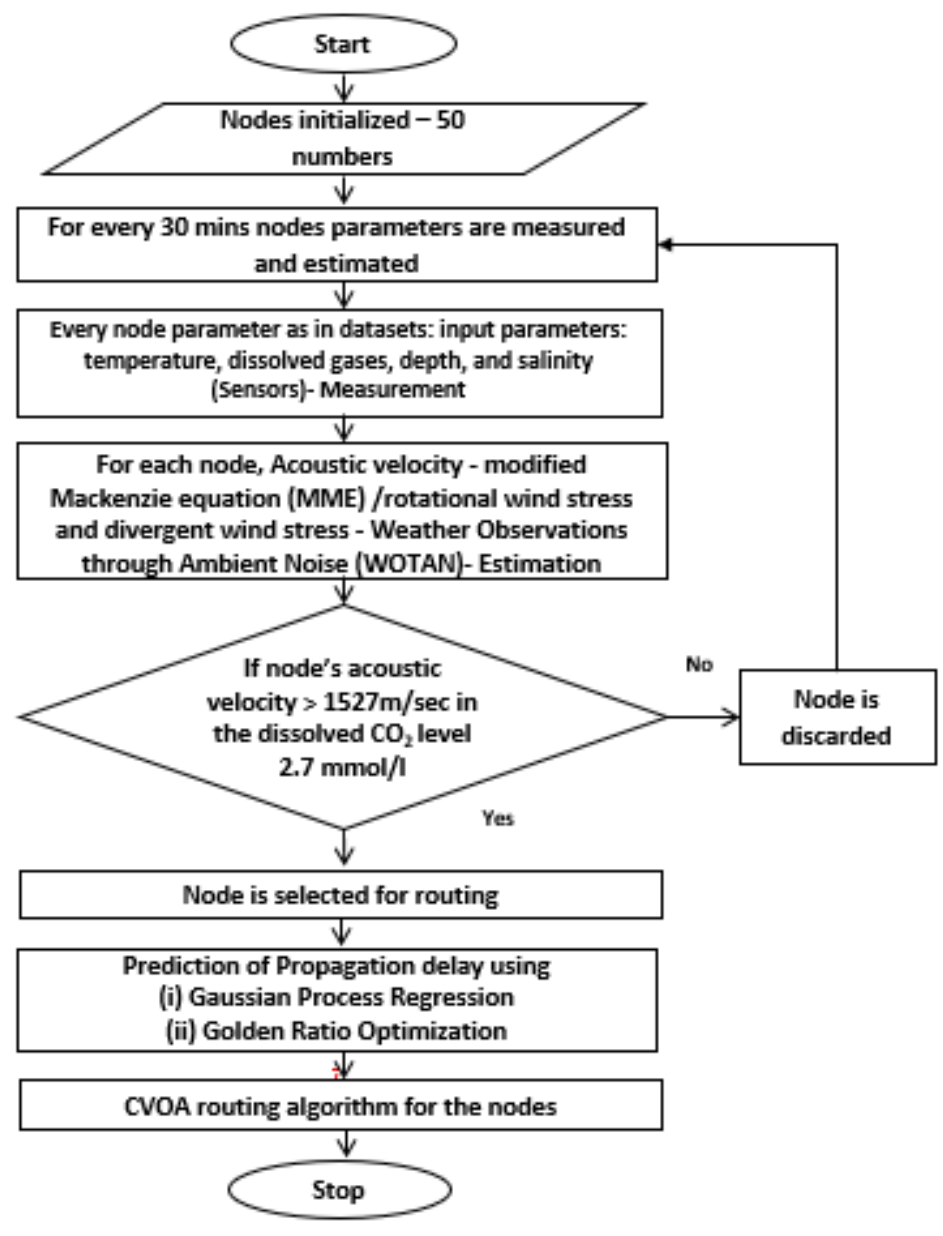

Figure 7

Routing using GPR, GROM and CVOA 


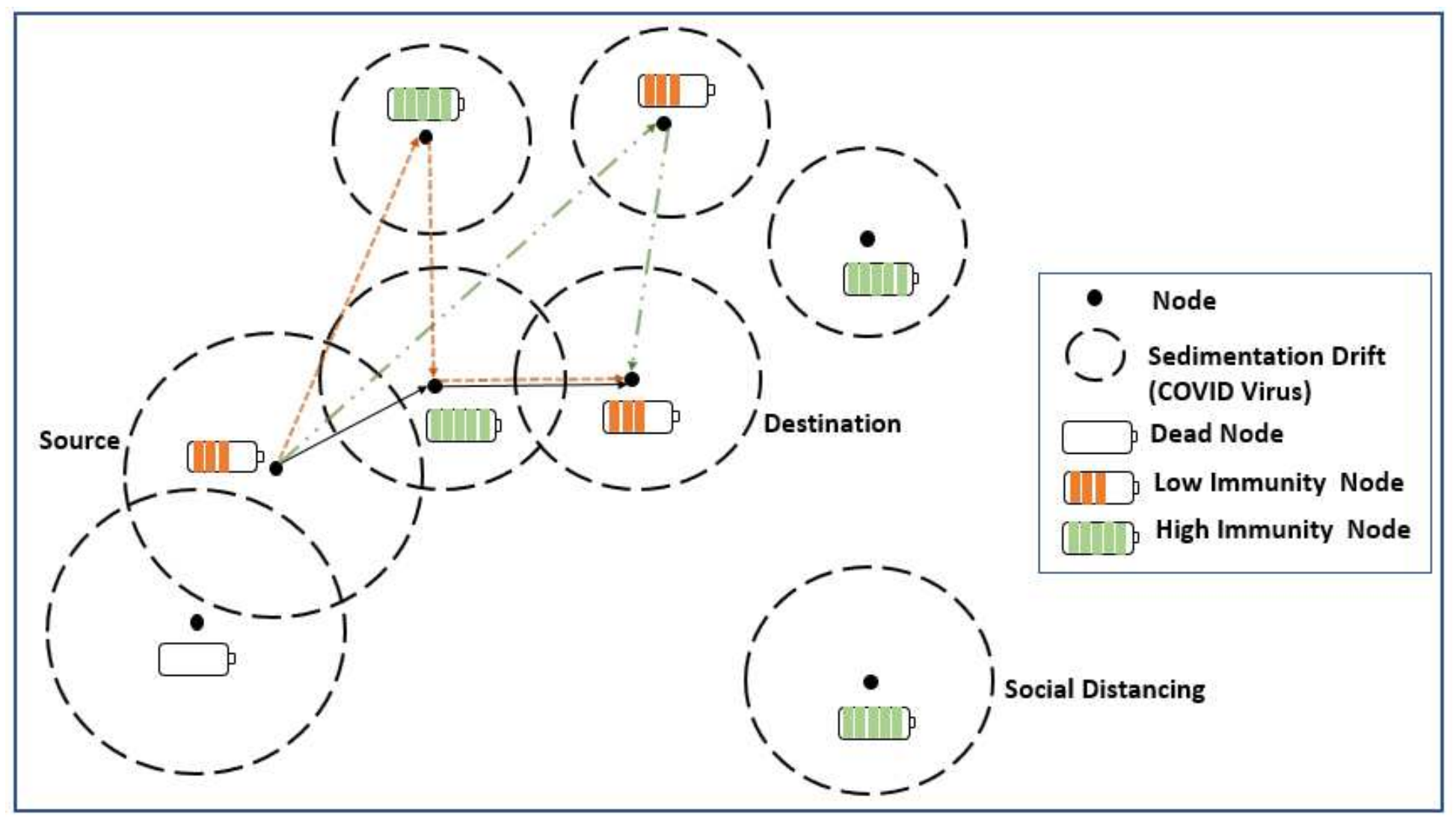

Figure 8

CVOA in UASN for routing

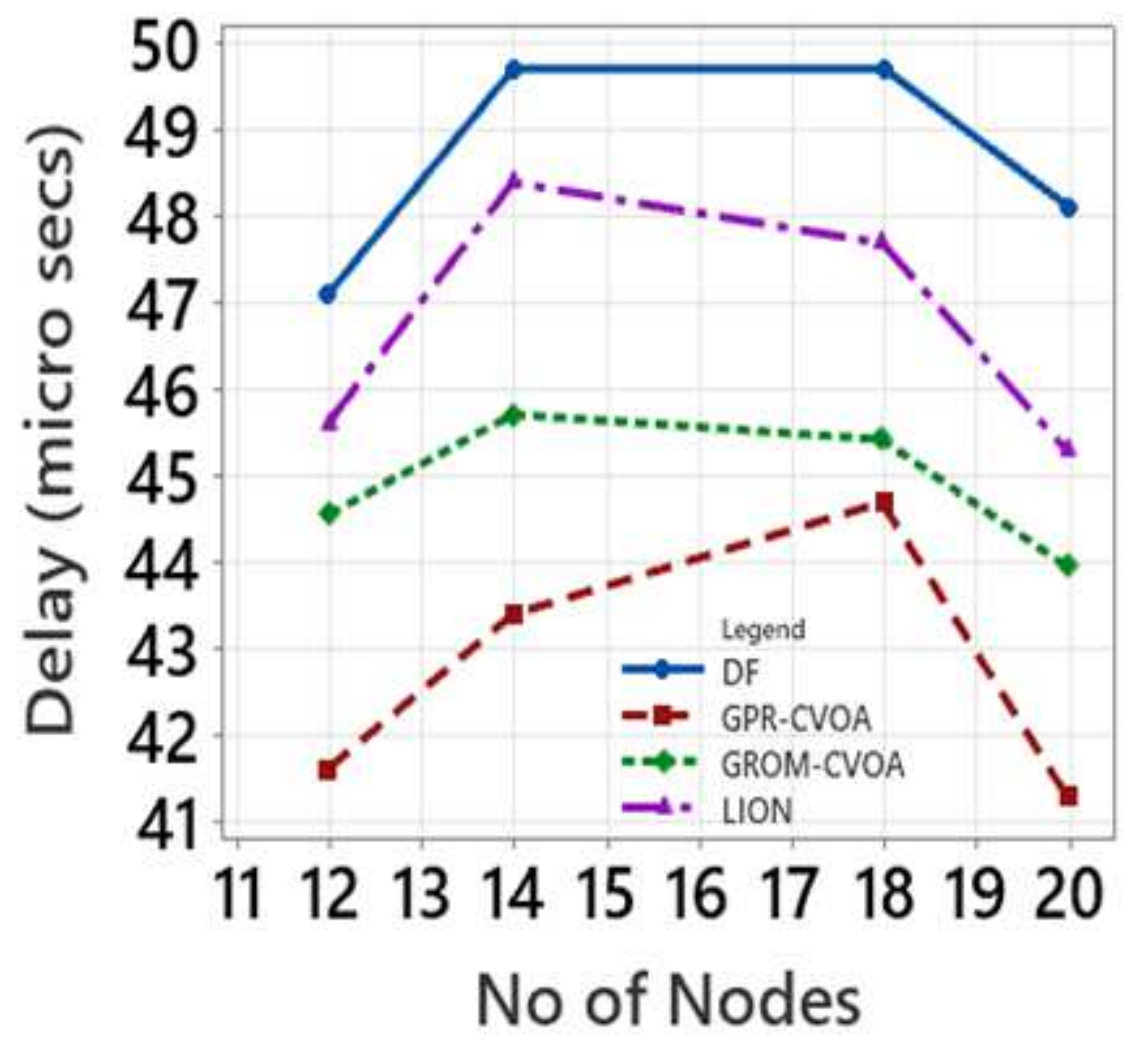

Figure 9 
Delay Vs Number of Nodes

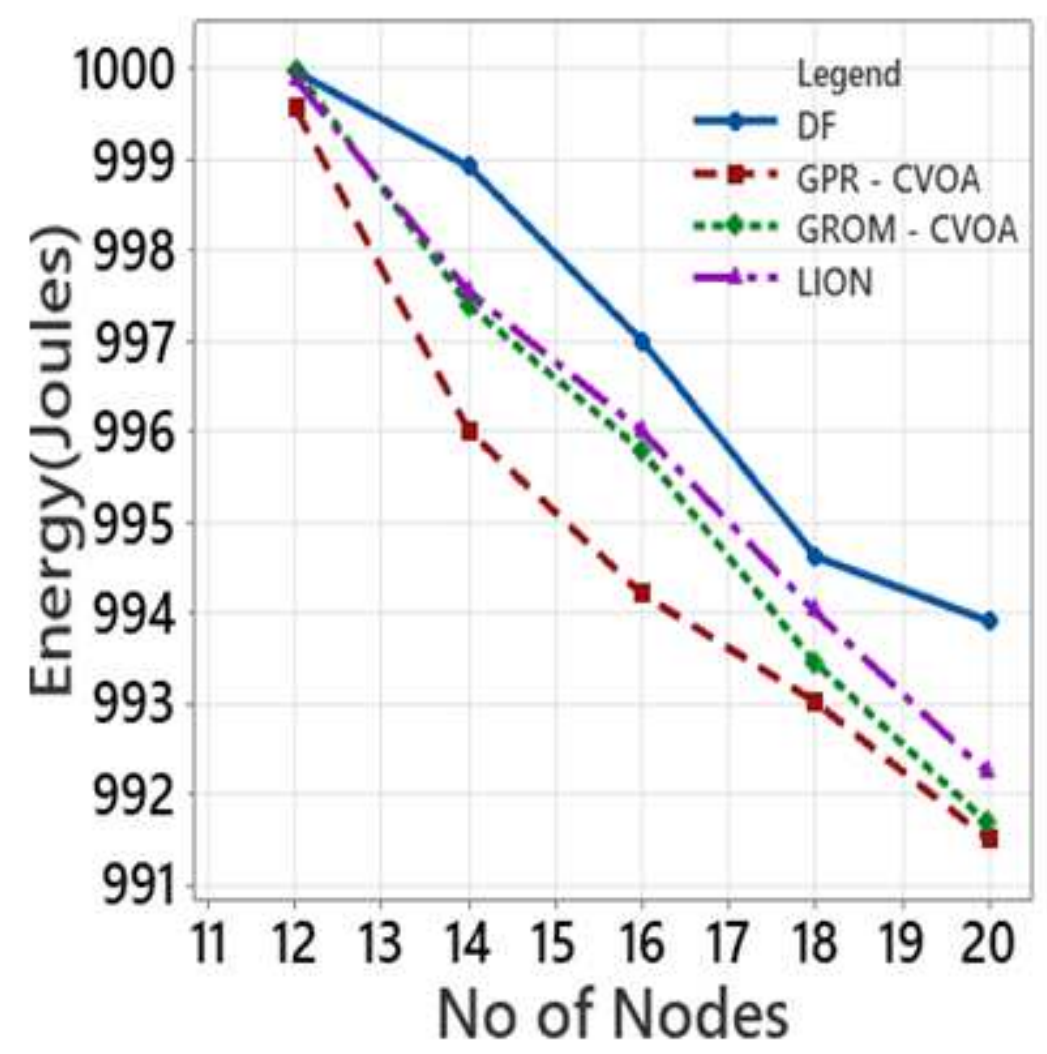

Figure 10

Energy Vs Number of Nodes

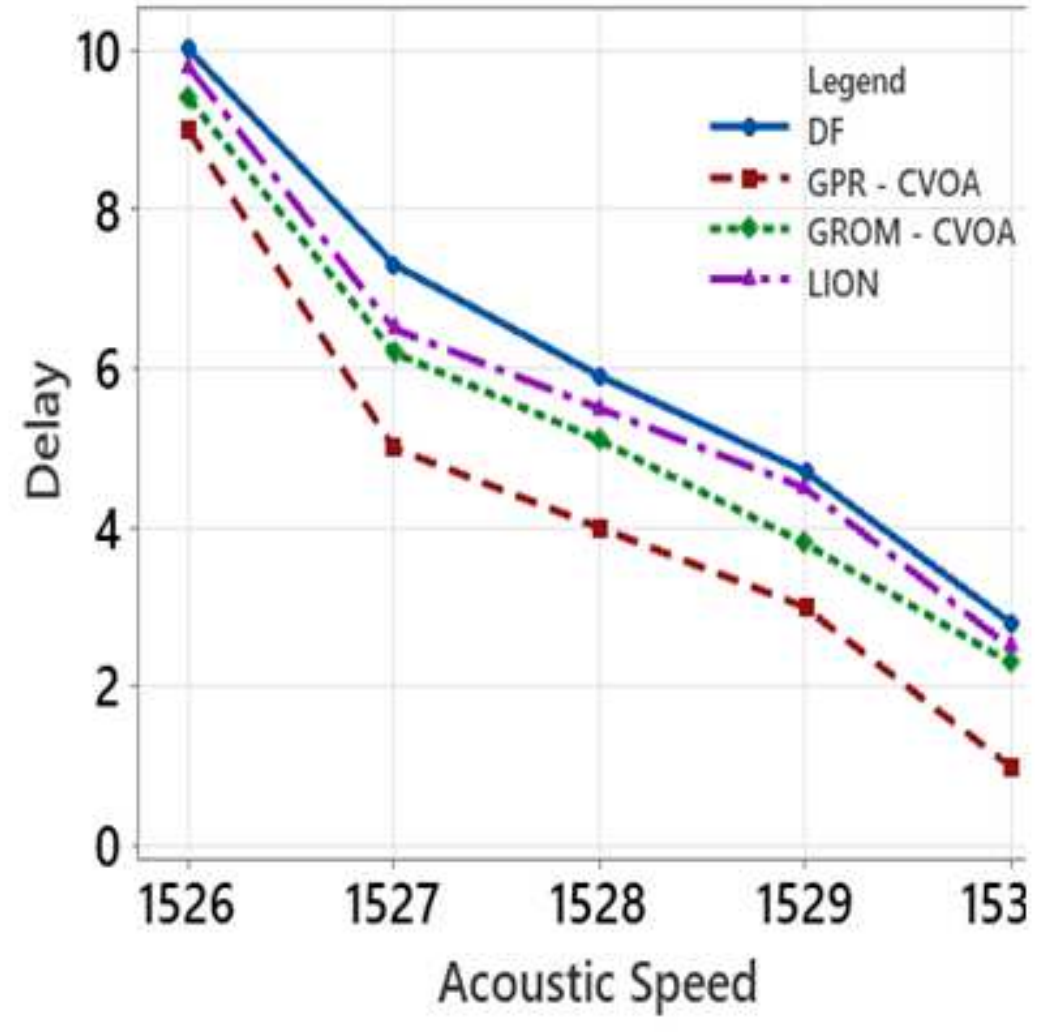


Figure 11

Acoustic Speed Vs Delay

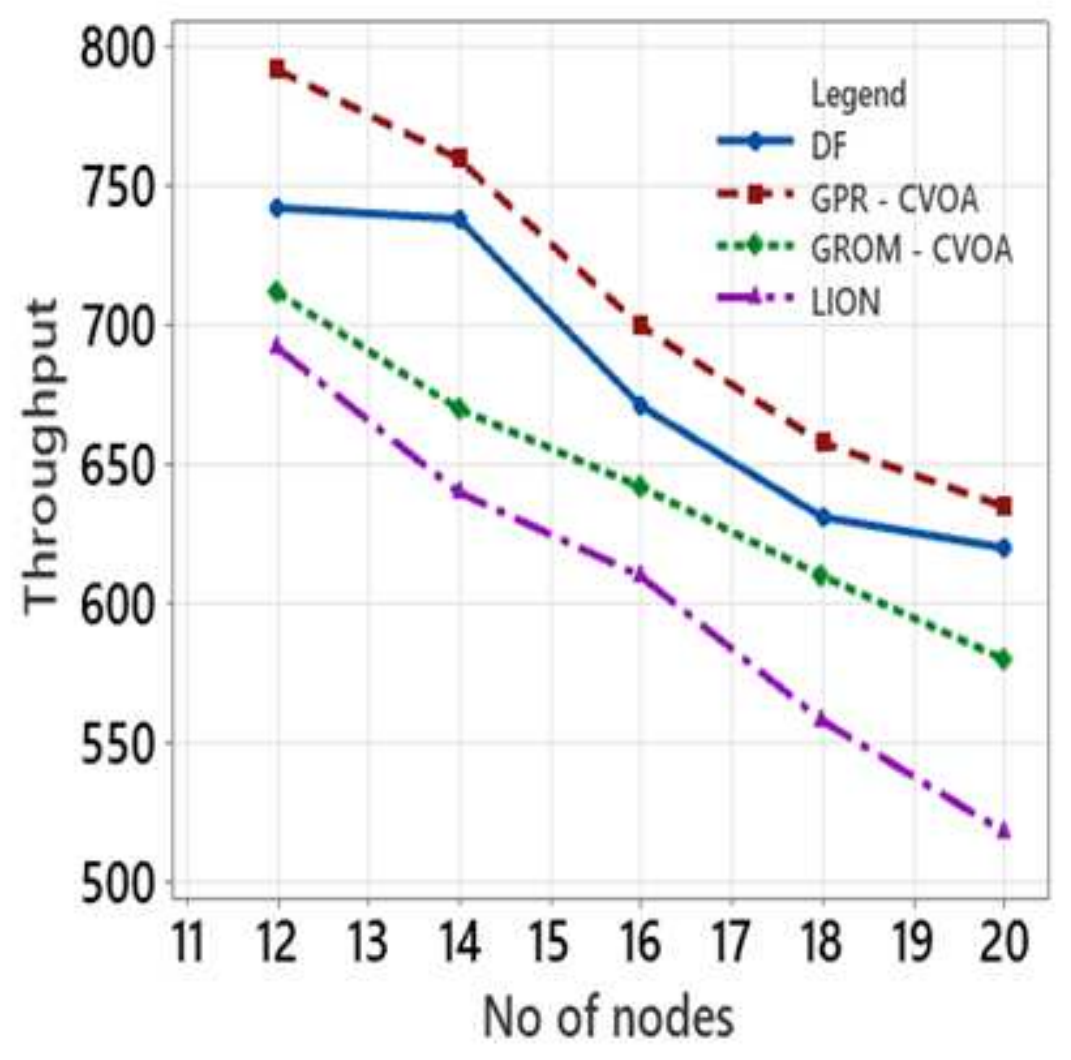

Figure 12

Throughput Vs Number of Nodes 


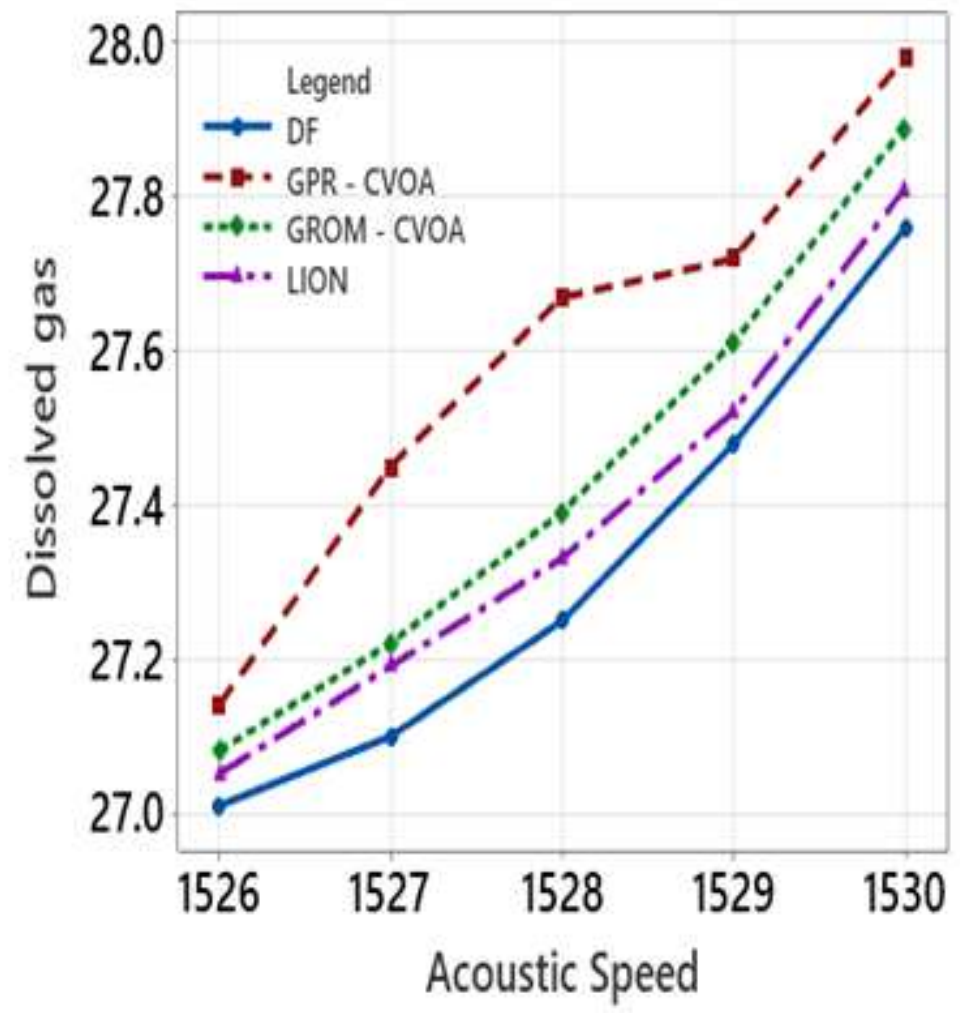

Figure 13

Dissolved gas Vs Acoustic Speed

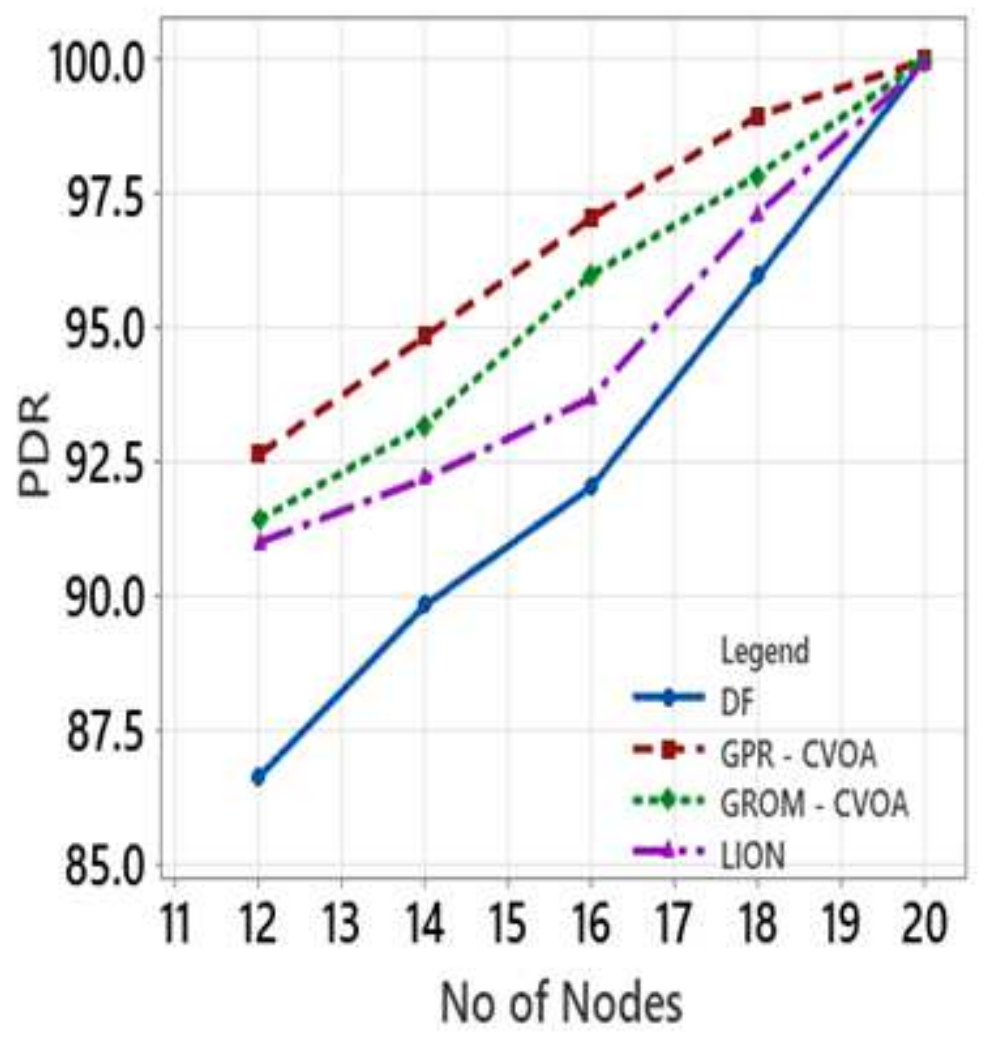

Figure 14 
PDR Vs Number of Nodes

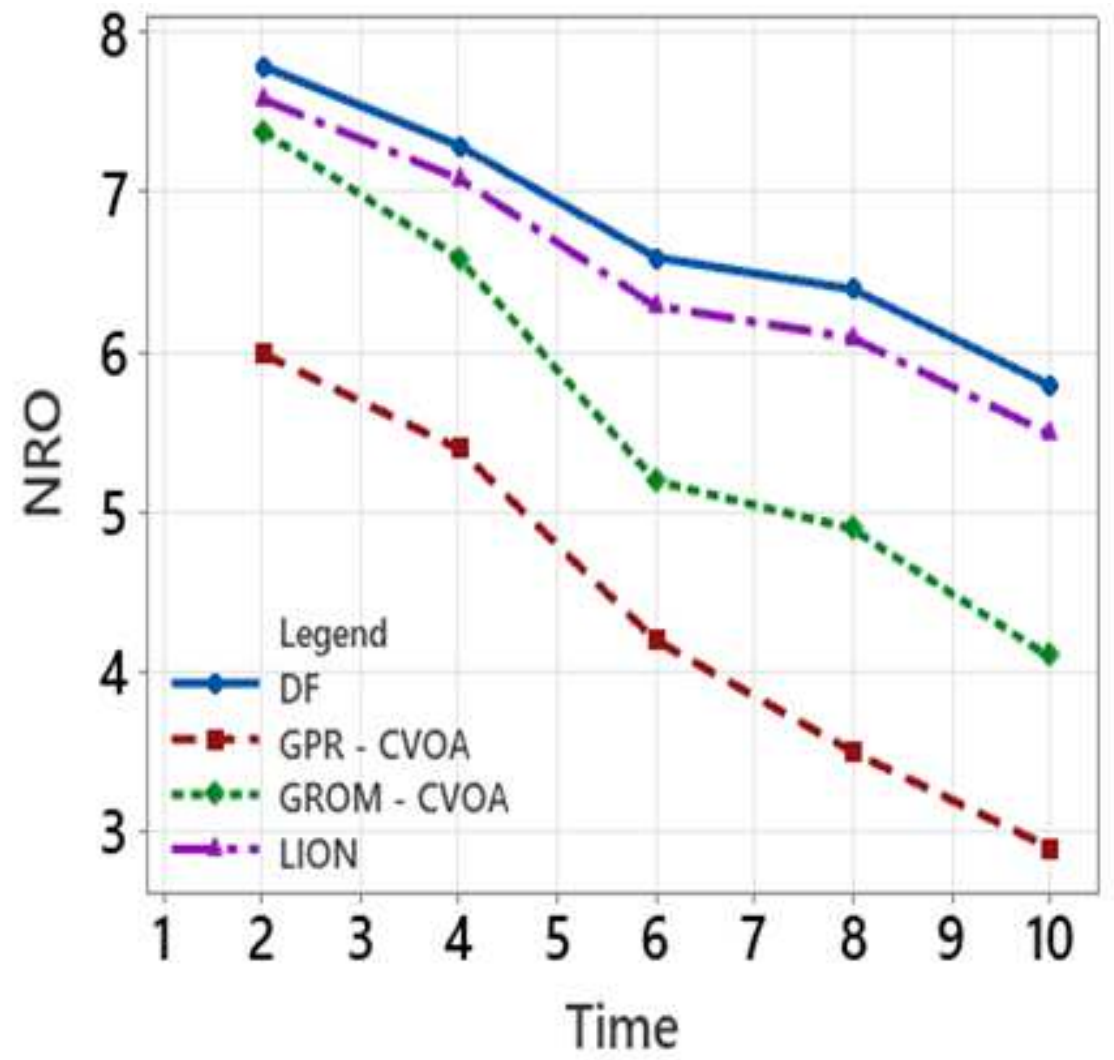

Figure 15

NRO Vs Time (millisecs)

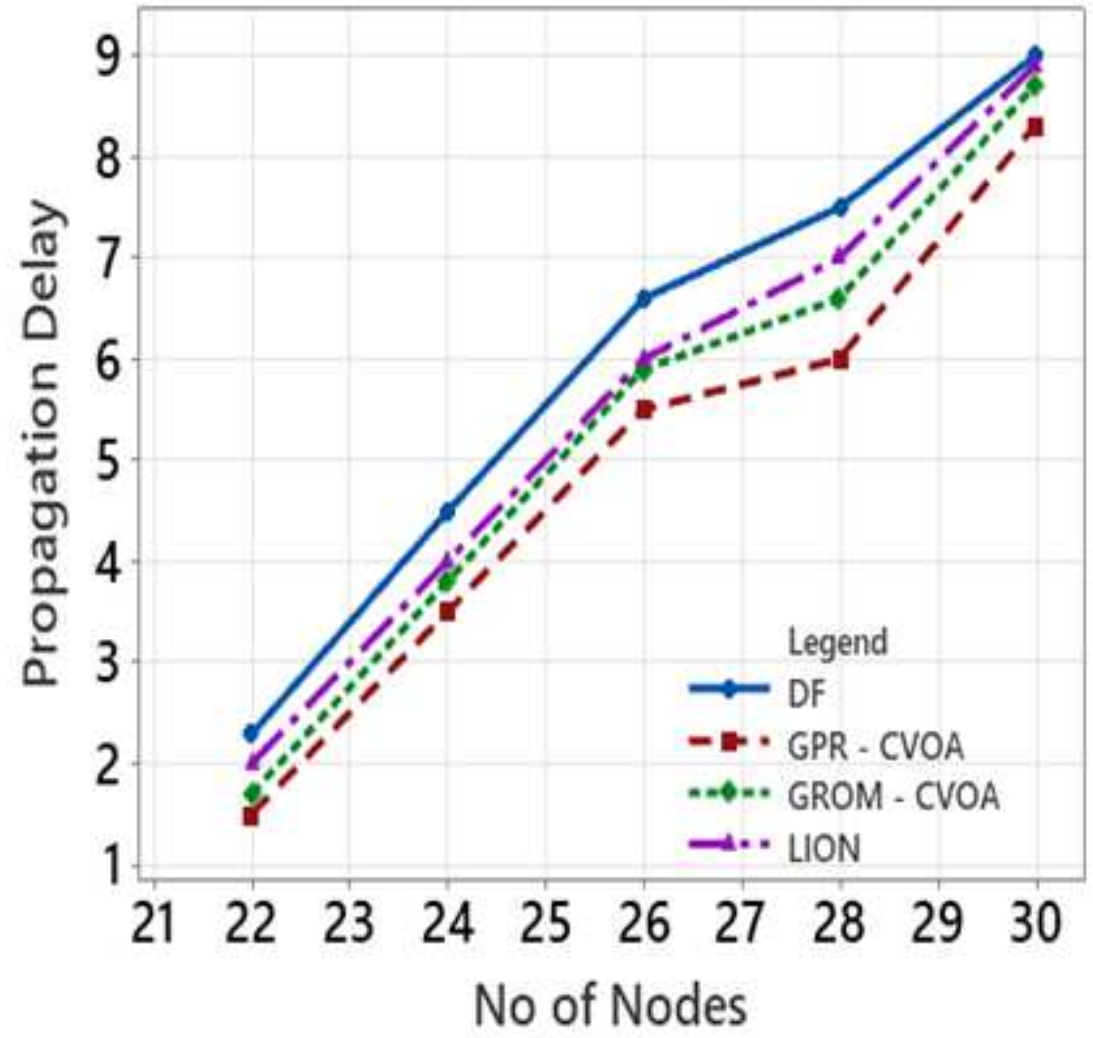


Figure 16

Propagation Delay vs Number of Nodes in the presence of Rotational Wind

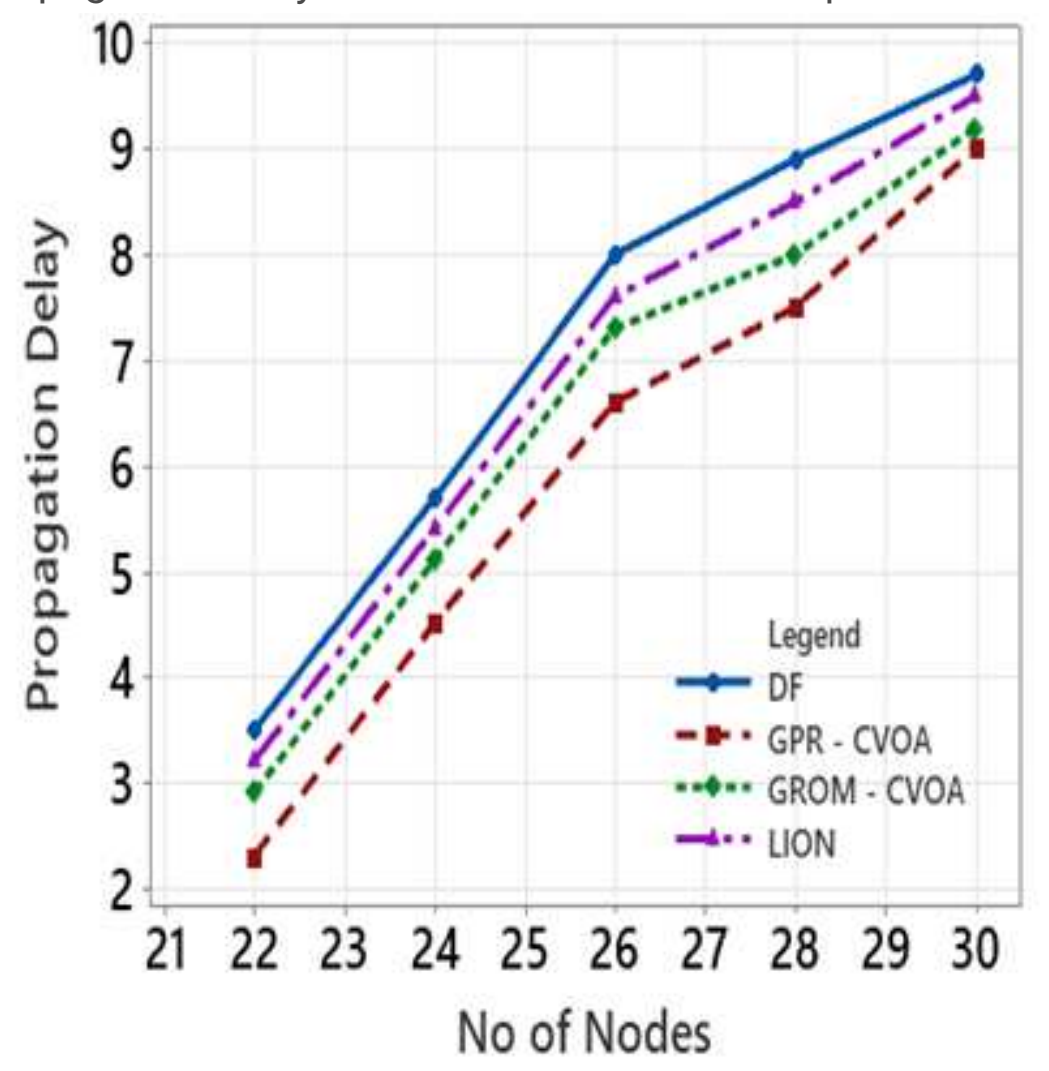

Figure 17

Propagation Delay vs Number of Nodes in the presence of Divergent Wind 